\title{
GMP-grade a-TEA lysine salt: a 28-Day oral toxicity and toxicokinetic study with a 28-Day recovery period in Beagle dogs
}

\author{
Bella S. Guerrouahen ${ }^{1}$, Tobias Hahn², Zefora Alderman², Brendan Curti ${ }^{2}$, Walter Urba ${ }^{2}$ and Emmanuel T. Akporiaye ${ }^{1,2^{*}}$
}

\begin{abstract}
Background: Alpha-tocopheryloxyacetic acid ( $\mathrm{a}-\mathrm{TEA}$ ) is a semi-synthetic derivative of naturally occurring vitamin E (alpha-tocopherol) that can be delivered via an oral route. Preclinical in vitro and in vivo data demonstrated that a-TEA is a potent anti-tumor agent with a safe toxicity profile in mice. We report a comprehensive study to evaluate the toxokinetics of good manufacturing practice (GMP)-grade a-TEA in dogs after daily oral administration for 28 days, followed by a 28-day recovery period.

Methods: Male and female beagle dogs received capsules of a-TEA Lysine Salt at doses of 100, 300, $1500 \mathrm{mg} / \mathrm{kg} / \mathrm{day}$. a-TEA plasma levels were determined by high-performance liquid chromatography (HPLC) with mass spectrometric detection. During the treatment, animals were observe for clinical signs, food consumption, body weight, and subjected to ophthalmoscopic, and electrocardiographic assessments. At the end of the dosing period, blood was taken and toxicokinetic analyses and histopathology assessments were performed when animals were necropsied.
\end{abstract}

Results: Our findings showed that there was no a-TEA-related mortality or moribundity. At the highest dose, increases in white blood cells and fibrinogen levels were observed. These levels returned to normal at the end of the recovery period. Histopathological evaluation of major organs revealed no significant lesions related to a-TEA-treatment.

Conclusion: We demonstrate that for designing clinical trials in patients, the highest non-severely toxic dose (HNSTD) of a-TEA is $1500 \mathrm{mg} / \mathrm{kg} /$ day in Beagle dogs and this data informed the design of dose-escalation studies of a-TEA in patients with advanced cancer.

Keywords: Cancer therapy, Vitamin analog, a-TEA lysine salt, Toxicokinetic, Pharmacokinetics, Non-rodent

\section{Background}

Alpha-tocopheryloxyacetic acid $(\alpha-T E A)$ is a semi-synthetic, non-hydrolysable ether derivative of vitamin E. Structurally, $\alpha$-TEA differs from vitamin $E$ by the replacement of the hydroxyl group at the carbon number 6 of the phenolic ring of the chroman head with an acetic acid residue linked by an ether bound [1]. $\alpha$-TEA is a cytotoxic drug that induces tumor cell death through targeting the mitochondria and by modulation of apoptosis and survival

\footnotetext{
* Correspondence: eakporiaye@sidra.org

'Sidra Medical and Research Center, Experimental Biology Division - Research, PO Box 26999, Doha, Qatar

${ }^{2}$ Laboratory of Tumor Immunology and Therapeutics, Earle A. Chiles Research Institute, Robert W. Franz Cancer Research Center, Providence Portland Medical Center, 4805 NE Glisan St. 2N35 Portland, OR, USA
}

pathways [2]. The in vivo anti-tumor activity of $\alpha$-TEA has been reported in several pre-clinical tumor models [1], and is partially dependent on a T-cell-mediated immune response [3-6]. Although $\alpha$-TEA has been evaluated as an anti-cancer agent in numerous pre-clinical tumor models [1, 7-10], efforts to translate these findings into human clinical trials are lacking. The goal of this study was to conduct a dose-escalation evaluation in an appropriate non-rodent animal species as required by the United States Food and Drug Administration (U. S. FDA) to obtain relevant toxico- and pharmaco-kinetic information in preparation for a first-inhuman trial to evaluate the safety and tolerability of $\alpha$ TEA in patients with advanced cancer. $\alpha$-TEA lysine salt 
was administered at increasing doses of 100,300 and $1500 \mathrm{mg} / \mathrm{kg}$ to male and female beagle dogs for $28 \mathrm{con}$ secutive days, and then observed for 28 days after the last $\alpha$-TEA dose. Complete measurements of body weight and food consumption were conducted over the treatment period. Ophthalmologic and electrocardiographic observations were assessed and clinical pathology evaluated. In this dose escalation study utilizing the lysine salt of $\alpha$ TEA, we demonstrated that daily administration of $\alpha$-TEA was not toxic at $1500 \mathrm{mg} / \mathrm{kg}$ body weight. The findings from this comprehensive and observational study to evaluate the pharmacology and toxicology of the $\alpha$ TEA (lysine salt) in beagle dogs formed the basis for initiating a first-in-human clinical trial in patients with advanced cancer that is ongoing (NCT 02192346).

\section{Methods}

\section{Preparation of a-Tocopheryloxyacetic acid lysine salt in} capsules

In the process of manufacturing $\alpha$-TEA free acid for pre-clinical development, the contract Commercial Research Organization (Ricerca Biosciences, LLC, Concord $\mathrm{OH})$ made the observation that during the initial scaleup, the free acid exhibited liquid crystal properties. Therefore, a salt screen was performed, which led to the identification and selection of the lysine salt. $\alpha$-TEA lysine salt ( $\alpha$-TEA LS) was synthesized (Fig. 1 ), using a modification of a previously described procedure [9]. Briefly, $\alpha$-TEA LS was prepared by reacting alpha-D- tocopherol with ethyl bromoacetate to form the ethyl ether intermediate. The ethyl ether intermediate was then reacted with potassium hydroxide to form $\alpha$-TEA free acid. The lysine salt was formed by adding aqueous lysine solution to a solution of $\alpha$-TEA in isopropyl alcohol. The lysine salt with its empirical formula of $\mathrm{C}_{37} \mathrm{H}_{66} \mathrm{~N}_{2} \mathrm{O}_{6}$ and its molecular weight of $634.93 \mathrm{~g} / \mathrm{mol}$ is a stable crystalline off-white powder. The dose concentration analysis was performed during the study and the test material was stable. Capsules for oral dose administration were prepared at least once weekly with appropriate amounts of bulk $\alpha$-TEA placed into gelatin capsules (Pharmatek LLC, San Diego CA). Oral administration is the planned route of administration in humans. The dose of the drug is expressed as free acid using a correction factor of 1.3 to reflect salt content (calculated as ratio of lysine salt/free acid molecular weight).

\section{Animal studies}

Initial studies in mice were conducted to assess the efficacy of the lysine salt form of $\alpha$-TEA against established tumors. Treatment of the animals (including but not limited to all husbandry, housing, and feeding conditions and euthanasia) was conducted in accordance with The Animal Welfare Act (Public Law 89-544) and the guidelines recommended in Guide for the Care and Use of Laboratory Animals (National Academy Press, Washington DC, 2011). The protocols and procedures involving the care and use of animals in

(a)<smiles>Cc1c(C)c2c(c(C)c1O)CCC(C)(CCCC(C)CCCC(C)CCCC(C)C)O2</smiles>

(b)<smiles>Cc1c(C)c2c(c(C)c1OCC(=O)O)CCC(C)(CCCC(C)CCCC(C)CCCC(C)C)O2</smiles>

(c)

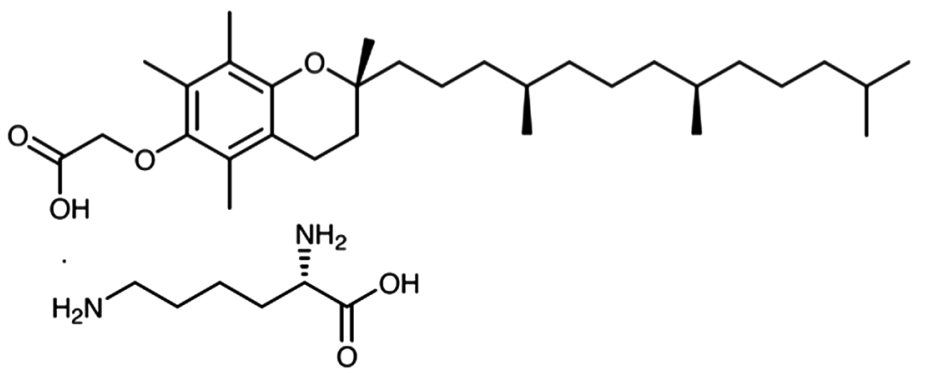

Fig. 1 Chemical structure of a-Tocopherol, a-Tocopheryloxy Acetic Acid (a-TEA), and a-tocopheryloxy acetic acid Lysine Salt (a-TEA LS). a a-Tocopherol. Molecular Weight $(M W)=430.69$. b a-Tocopheryloxy Acetic Acid (a-TEA). MW=488. c a-TEA Lysine Salt. MW =634.93 
the study were reviewed and approved by the Earle A. Chiles Research Institute and Ricerca Institutional Animal Care and Use Committees (IACUC).

$\alpha$-TEA was formulated in mouse chow and delivered to mice bearing established 4 T1 mammary tumors, as we previously described. This anti-tumor study was followed by a toxicology study. For this purpose, both the $\alpha$-TEA free acid and $\alpha$-TEA LS forms were administered to naïve mice intravenously or by oral gavage. A total of 44 Beagle dogs (Canis familiaris; were acquired by the contract CRO (Ricerca Biosciences) from Marshall BioResources, North Rose, NY) for the study. Animals were as uniform in age as possible; dogs were pre-pubertal to young adults, at least 8 months of age (range of 7.8 to 8.3 months of age) and weighed between 4 and $8 \mathrm{~kg}$ at the start of dose administration. The frequency of administration was designed to mimic the regimen intended for human trial. $\alpha$-TEA LS was administered in gelatin capsules once daily to 22 male and 22 female dogs at 100,300 and $1500 \mathrm{mg} / \mathrm{kg}$ for 28 consecutive days. The amount for each animal was based on the most recent body weight. Control animals received equal number of empty gelatin capsules as the high dose animals. Animals were observed for viability and clinical signs, daily food consumption, body weight (once weekly or twice for high dose animals). Group assignment and dose level are shown in the Table 1. Clinical pathology, ophthalmic and electrocardiography examinations were done at the pre-dose and after the terminal dose. Eight dogs from each group (4 females and 4 males) were necropsied at the end of the dosing period (Day 29) or at the end of the recovery period (Day 57). Animals were fasted overnight prior to scheduled blood test or necropsy. When necropsied, organs were collected and preserved in $10 \%$ neutral-buffered formalin with the exception of the testes, epididymides, and eyes; which were fixed in Modified Davidson's Solution for 24 to $48 \mathrm{~h}$, water washed, and then transferred to $10 \%$ neutral-buffered formalin for storage. Histopathology assessments were performed. Bone marrow smears were stained with Wright-Giemsa before cytology evaluation.

\section{Toxicokinetics studies}

Toxicokinetics studies were performed by Celerion Inc., a commercial research organization based in Lincoln, Nebraska, USA. Toxicokinetic parameters were studied on blood collected from the jugular vein of the animal $(1 \mathrm{~mL})$ on day 1 and on the last day of treatment. Blood samples were collected into $\mathrm{K}_{3}$ EDTA-containing tubes at $1,2,4,8$, and $24 \mathrm{~h}$ following the first $\alpha$-TEA administration. Following the last administration (day 28), blood collection for recovery animals was scheduled as follows: 2, 8, 24, 72, and $168 \mathrm{~h}$. For the control animals (group 1 ), only the 8 -hour sample was collected. The chosen time points were based on kinetic data gathered from pilot toxicity studies conducted in mice. Recovered plasma was stored at $-70{ }^{\circ} \mathrm{C}$ until analysis.

\section{Data collection}

Animal data, such as observations, body weights, food consumption, clinical pathology values, necropsy findings, and organ weights, were collected and reported electronically using Provantis ${ }^{\mathrm{Tw}}$, Version 8 (Instem LSS Ltd. Staffordshire, UK). Urine was collected and analyzed by a Clinitek Atlas Urinalysis System. Data from the examination of urine sediment were entered directly into Provantis $^{\mathrm{TM}}$. Toxicokinetics data were collected and stored in electronic notebook system Labnotes ${ }^{\mathrm{TM}}$ Client 1.18.

\section{Sample preparation and High Performance Liquid Chromatography (HPLC) analysis}

Systemic levels of $\alpha$-TEA were determined using HPLC with mass spectrometry. Bioanalytical data were obtained from Celerion, Inc according to the SOPs written based on the GLP principles. Briefly, an aliquot of the extracted dog plasma was analyzed by an HPLC equipped with an AB SCIEX API $4000^{\mathrm{TM}}$ triple quadrupole mass spectrometer using an ESI source. Negative ions were monitored in the multiple reaction-monitoring (MRM) mode. Quantitation was determined using a weighted linear regression analysis (1/concentration $\left.{ }^{2}\right)$ of peak area ratios of the analyte and internal standard. The area under the plasma concentration-time curves, peak

Table 1 Group assignment and dose levels

\begin{tabular}{|c|c|c|c|c|c|c|}
\hline \multirow[t]{2}{*}{ Group } & \multirow[t]{2}{*}{ Number of animals (M/F) } & \multirow[t]{2}{*}{ Test article } & \multirow[t]{2}{*}{ Nominal dose level (mg/kg/day) } & \multirow[t]{2}{*}{ Actual dose level ${ }^{a}$} & \multicolumn{2}{|c|}{ Number of animals for necropsy (M/F) } \\
\hline & & & & & Terminal (Day 29) & Recovery (Day 57) \\
\hline 1 & $6 / 6$ & Vehicle & 0 & 0 & $4 / 4$ & $2 / 2$ \\
\hline 2 & $4 / 4$ & $a-T E A$ & 100 & 130 & $4 / 4$ & - \\
\hline 3 & $6 / 6$ & $a-T E A$ & 300 & 390 & $4 / 4$ & $2 / 2$ \\
\hline 4 & $6 / 6$ & a-TEA & 1500 & 1950 & $4 / 4$ & $2 / 2$ \\
\hline
\end{tabular}

${ }^{\mathrm{a}}$ Dose levels have been corrected for lysine salt using a correction factor of 1.3

A total of 44 Beagle dogs (22 males and 22 females) received a-TEA lysine salt at different concentrations $(0,100,300,1500 \mathrm{mg} / \mathrm{kg})$. Eight dogs from each group ( 4 females and 4 males) were necropsied at the end of dosing period (Day 29) or at the end of recovery period (Day 57) 
plasma concentration, time to achieve peak plasma concentration, and plasma terminal half-life (AUC, $\mathrm{C}_{\max }, \mathrm{T}_{\max }$, and $\mathrm{T}_{1 / 2}$ ) were determined using WinNonlin Version 6.2 (Pharsight, Mountain View CA), operating as a validated software system.

\section{Statistical analysis}

For comparative statistics, data through the Day 29 termination were evaluated using the Levene Test for homogeneity of variances and the Shapiro-Wilks Test for normality of distributions, with significance at $p \leq 0.05$. Data determined to be homogeneous and of normal distribution were evaluated for analysis of variance (ANOVA). If the ANOVA verified significance at $p \leq 0.05$, pairwise comparisons of each treated group with the control group were made using a parametric test, Dunnett $t$-test, to identify statistical differences $(p \leq 0.05)$. Data determined to be nonhomogeneous or of non normal distribution were evaluated using a Kruskal-Wallis Test for group factor significance. If significance $(p \leq 0.05)$ existed between groups, a nonparametric test, Wilcoxon with Bonferroni-Holm, was used to compare treated groups to the control group.

\section{Results}

Anti-tumor activity of a-TEA lysine salt

We first tested the anti-tumor activity of GMPmanufactured $\alpha$-TEA LS, by conducting experiments in BALB/c mice bearing established $4 \mathrm{~T} 1$ mammary tumors as previously described [11]. Tumor bearing mice received (i) control diet until 5 days post-tumor cell injection, and then were switched to $0.39 \% \alpha$ TEA salt diet; or (ii) $390 \mathrm{mg} / \mathrm{kg}$ body weight $\alpha$-TEA LS diet. After 60 days of tumor monitoring, we found that $\alpha$-TEA LS significantly inhibit tumor growth (Fig. 2a) and prolonged overall survival compared to control diet (Fig. 2b). Blood samples were collected from 3 naïve mice/group and systemic levels of $\alpha$-TEA were determined by HPLC with mass spectrometry detection in order to compare the pharmacokinetics and exposure of a single dose of $\alpha$-TEA LS with that of the (a)

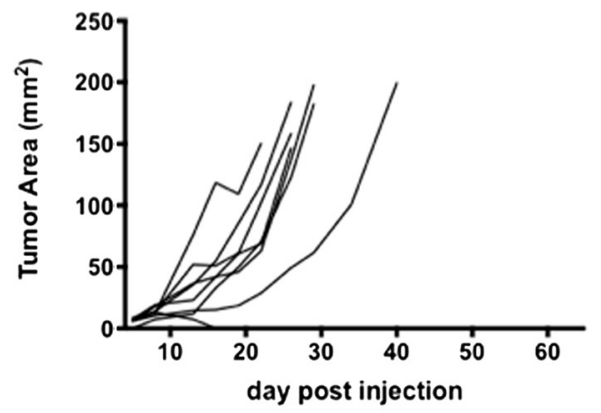

(b)

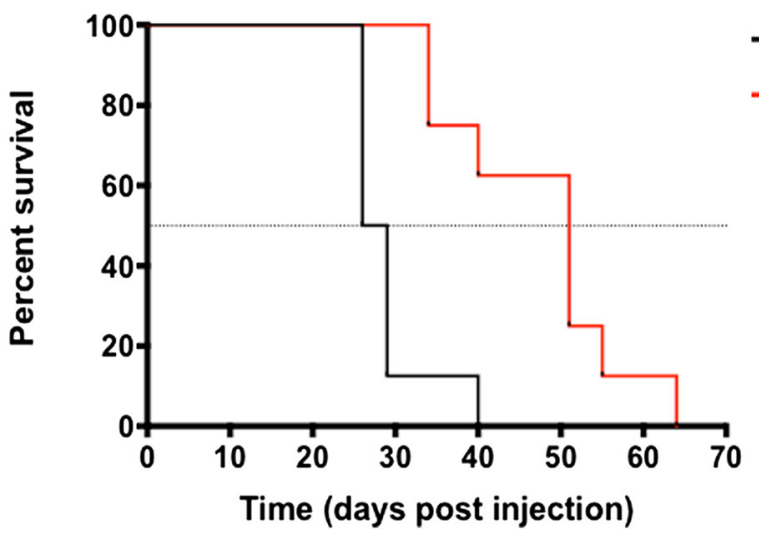

$\alpha$-TEA Lysine Salt

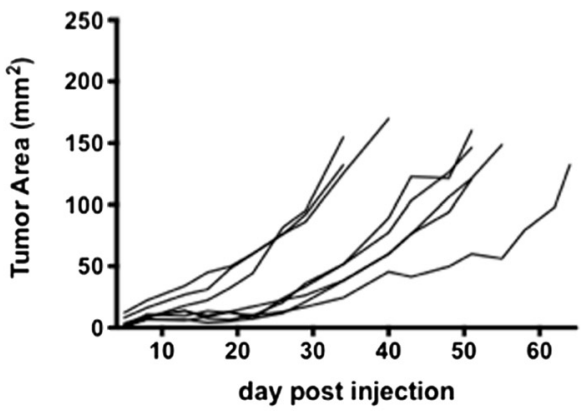

Fig. 2 Anti-tumor activity of a-TEA lysine salt. a Effect of dietary delivery of a-TEA lysine salt on primary tumor growth. BALB/c mice were injected with $5 \times 10^{4} 4 \mathrm{~T} 1$ tumor cells in the right mammary fat pad. When tumors became palpable ( 5 days post tumor cell injection) mice received $390 \mathrm{mg} / \mathrm{kg} /$ day a-TEA lysine salt diet (equivalent to $300 \mathrm{mg} / \mathrm{kg} /$ day a-TEA) and tumor growth was monitored. b Oral a-TEA lysine salt prolongs survival of mammary tumor-bearing mice. BALB/c with established $4 \mathrm{~T} 1$ mammary tumors (day $5, \sim 4-6 \mathrm{~mm}^{2}$ ) received oral a-TEA lysine salt diet (390 mg/kg bodyweight) or nutrient-matched control diet. Mantel-Cox analysis of survival of $n=8$ mice per group 
Mean ( \pm SD) Plasma $\alpha$-TEA Free Acid Concentrations Versus Time

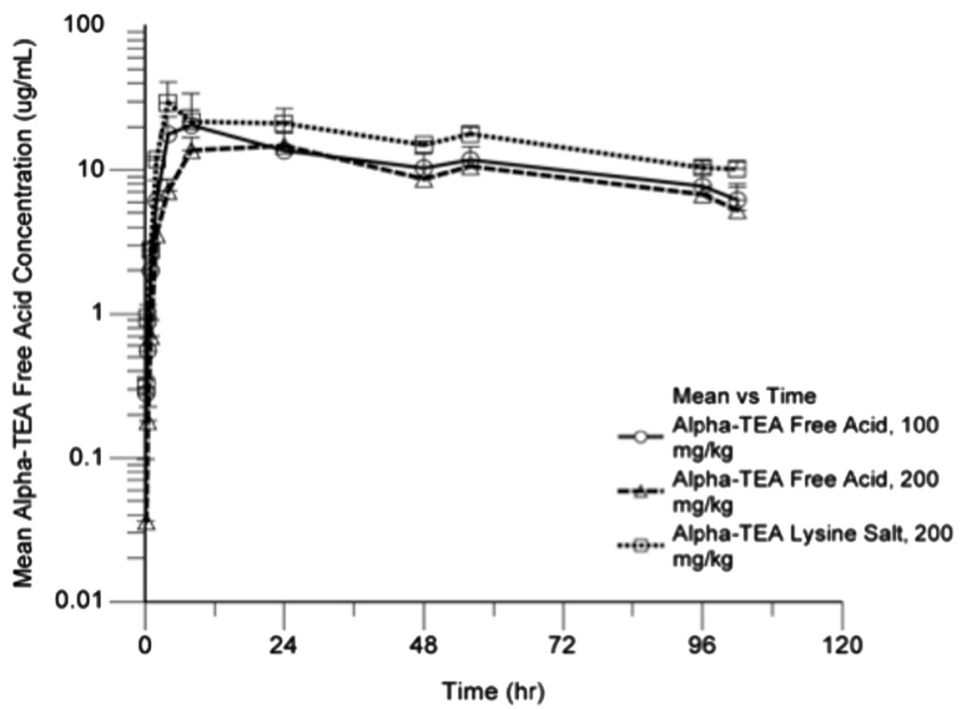

Fig. 3 Exposure of BALB/c mice to a single-dose of lysine salt or free acid a-TEA. a-TEA free acid (100 mg/kg) and a-TEA lysine salt (200 mg/kg) were administered by intravenous injection or oral gavage. Blood was collected from 3 mice/group at each time point and analyzed for a-TEA levels by high performance liquid chromatography (HPLC) with mass spectrometric detection (LC-MS/MS)

free acid. The free acid could only be administered by gavage because it was toxic when administered by intravenous injection. However, both routes of administration could be used with the lysine salt. As shown in Fig. 3 and Table 2, when $\alpha$-TEA was administered orally by gavage at the same dose level $(200 \mathrm{mg} / \mathrm{kg})$, exposure was higher with $\alpha$-TEA LS compared to $\alpha$ TEA free acid. The peak plasma concentration after administration $\left(C_{\max }\right)$ was 2 times higher with $\alpha$-TEA LS compared to the $\alpha$-TEA free acid (Fig. 3). We observed a saturation phenomenon reflected by a slight decrease in $C_{\max }$ following the increase in dose level from $100 \mathrm{mg} / \mathrm{kg}$ to $200 \mathrm{mg} / \mathrm{kg}$ of $\alpha$-TEA free acid (Table 2). Our data indicate that the time to reach the peak plasma concentration after administration $\left(\mathrm{T}_{\max }\right)$ is $4 \mathrm{~h}$ with $\alpha$-TEA LS versus $24 \mathrm{~h}$ for $\alpha$-TEA free acid. The elimination half-lives was similar between 100 and $200 \mathrm{mg} / \mathrm{kg} \alpha$-TEA free acid and $200 \mathrm{mg} / \mathrm{kg} \alpha$-TEA lysine salt (54.6/50.2/54.6 $\mathrm{h}$ respectively).

\section{Repeat daily dosing of a-TEA Lysine Salt did not cause gross toxicity}

Several experiments were performed to evaluate the pharmacology and toxicology profile of repeated dosing of GMP-grade $\alpha$-TEA LS in Beagle dogs, over a period of 28 days followed by a 28 -day recovery period. Dose levels were chosen based on available data in mice [11] and from a preliminary dog tolerability study. Once daily, $\alpha$-TEA salt was administered in gelatin capsules to male and female Beagle dogs at 100, 300 and $1500 \mathrm{mg} / \mathrm{kg}$ body weight for 28 consecutive days. Clinical signs were limited to fecal changes (decreased/discolored) and emesis in $1500 \mathrm{mg} / \mathrm{kg}$ animals on several occasions following start of dose administration and continuing until the start of recovery period. The decreased feces coincided with transient decreases in food consumption noted in several animals, and persisted upon continued dosing. $\alpha$-TEA effects on food consumption at $1500 \mathrm{mg} / \mathrm{kg}$ were also reflected in the slightly decreased in body weight gain in

Table 2 Effect of a single dose of a-TEA lysine salt or free acid on serum pharmacokinetics parameters

\begin{tabular}{lllllll}
\hline Test article & Dose level $(\mathrm{mg} / \mathrm{kg})$ & $\mathrm{T}_{\max }(\mathrm{hr})$ & $\mathrm{T}_{1 / 2}(\mathrm{hr})$ & $\mathrm{C}_{\max }(\mathrm{ug} / \mathrm{mL})$ & $\mathrm{AUC}_{0-24}\left(\mathrm{hr}{ }^{*} \mathrm{ug} / \mathrm{mL}\right)$ & $\mathrm{AUC} \mathrm{C}_{0-\infty}\left(\mathrm{hr} /{ }^{*} \mathrm{ug} / \mathrm{mL}\right)$ \\
\hline a-TEA Free Acid & 100 & 8 & 54.6 & 20.4 & 1,180 & 1,670 \\
a-TEA Lysine Acid & 200 & 4 & 54. & 29.4 & 1,680 & 2,470 \\
a-TEA Free Acid & 200 & 24 & 50.2 & 14.7 & 1,020 & 1,400
\end{tabular}

a-TEA free acid (100 mg/kg) and a-TEA lysine salt $(200 \mathrm{mg} / \mathrm{kg}$ ) were administered by intravenous injection or oral gavage in BALB/c mice. Blood was collected from 3 mice/group at each time point and analyzed for a-TEA levels by HPLC with mass spectrometric detection (LC-MS/MS). The peak plasma concentration of a drug after administration $\left(C_{\max }\right)$, the time required to reach it $\left(T_{\max }\right)$, the time required for the concentration of the drug to reach half of its original value $\left(T_{1 / 2}\right)$, the area under the plasma drug concentration-time curve (AUC) were determined using WinNonlin Version 6.2 software system 
all $1500 \mathrm{mg} / \mathrm{kg}$ males during the second and third week of dose administration and in two of six females during the second week and all the $1500 \mathrm{mg} / \mathrm{kg}$ females during the third week of administration (Fig. 4a, b). Individual body weight gain decreases were present at 300 and $100 \mathrm{mg} / \mathrm{kg}$; however, overall group mean was comparable to controls and the changes were not considered toxicologically relevant. During the recovery period, the body weight gain in control and $\alpha$-TEA-treated animals was comparable (data not shown). There was no moribundity or mortality throughout the course of treatment. There were no $\alpha$-TEA-related ophthalmologic findings, but the $1500 \mathrm{mg} /$ $\mathrm{kg}$ group showed sinus bradycardia on electrocardiograms at day 27 for the males and day 26 for the females.
Clinical pathology (erythroid, leukocytes, coagulation parameters, serum chemistry and urinalysis) of a-TEAtreated animals

At the dosing level of 100,300 , and $1500 \mathrm{mg} / \mathrm{kg}$, no effect on erythroid or serum chemistry was observed. On day 29, white blood cell counts (neutrophils and lymphocytes) and fibrinogen levels were slightly higher in animals that received $1500 \mathrm{mg} / \mathrm{kg} \alpha$-TEA LS (in 2 of 4 males and 2 of 4 females) compared to their pre-dose level (Tables 3 and 4). Interestingly, at the end of the recovery period, no $\alpha$-TEArelated changes in coagulation or leukocyte parameters were recorded (Tables 5). On Day 29, evaluation of serum chemistry parameters showed that the mean chloride concentration (CL), mean blood urea nitrogen concentration
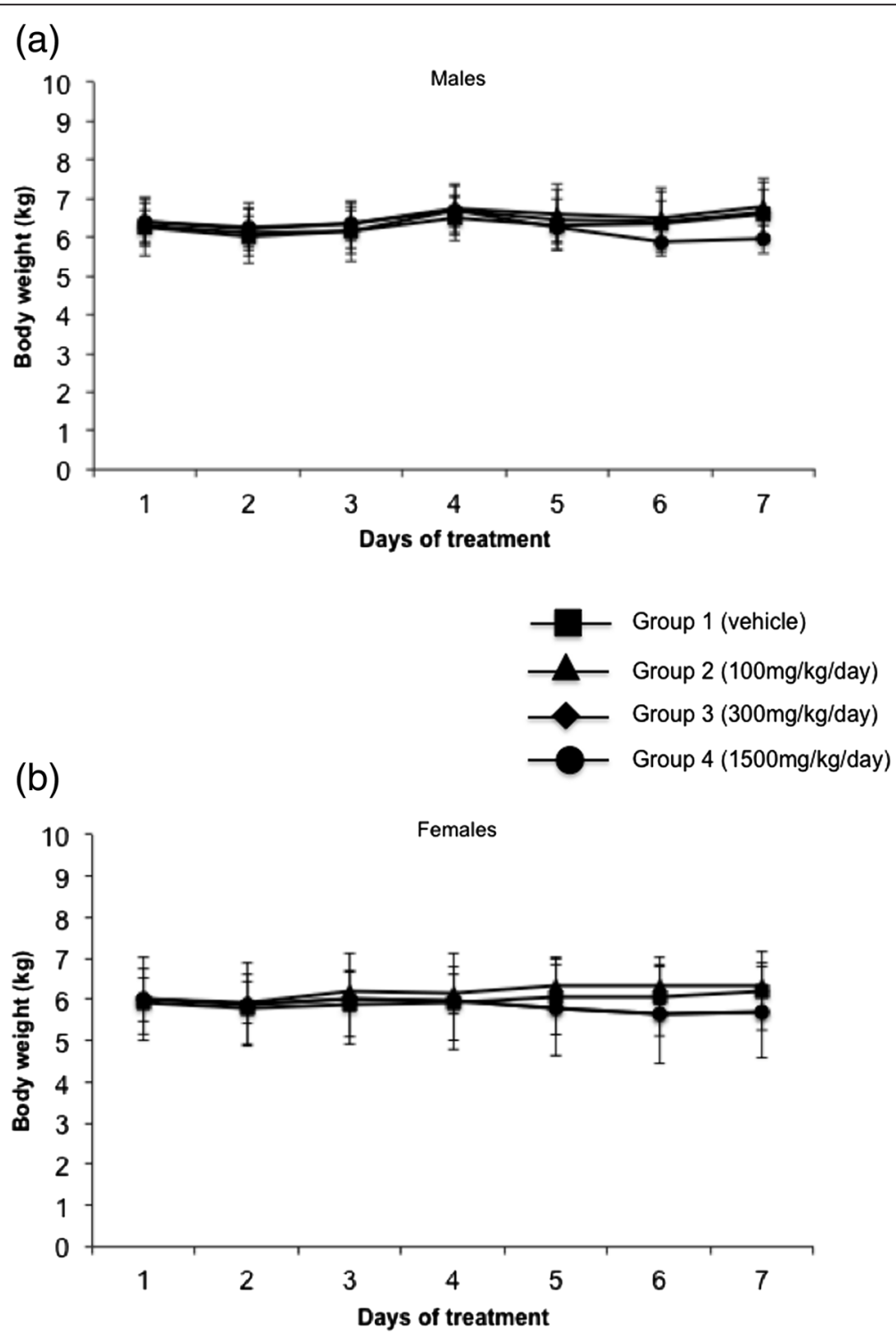

Fig. 4 Measure of body weights during 28-day dosing of a-TEA lysine salt. Male (a) and female dogs (b) received indicated doses of a-TEA lysine salt daily. Body weights were determined twice before the start of the treatment and every week following the start of the treatment. Data points represent mean body weight \pm SD 
Table 3 Mean blood cells and coagulation parameters in dogs 10 days prior to treatment

\begin{tabular}{|c|c|c|c|c|c|c|c|c|c|c|c|c|c|c|}
\hline & & \multicolumn{3}{|c|}{ Vehicle } & \multicolumn{3}{|c|}{100 mg/kg } & \multicolumn{3}{|c|}{300 mg/kg } & \multicolumn{3}{|c|}{$1500 \mathrm{mg} / \mathrm{kg}$} & \multirow[t]{2}{*}{ Unit } \\
\hline & & Mean & SD & N & Mean & SD & $\mathrm{N}$ & Mean & SD & N & Mean & SD & $N$ & \\
\hline \multirow[t]{21}{*}{ Males } & Erythrocytes & & & & & & & & & & & & & \\
\hline & Red blood cells & 6.315 & 0.485 & 6 & 6.418 & 0.316 & 4 & 6.523 & 0.211 & 6 & 6.74 & 0.416 & 6 & M/ul \\
\hline & Hemoglobin & 13.98 & 1.13 & 6 & 13.83 & 0.71 & 4 & 14.43 & 0.48 & 6 & 14.8 & 1.21 & 6 & $g / L$ \\
\hline & Hematocrit & 43.18 & 3.51 & 6 & 43.08 & 1.54 & 4 & 44 & 1.5 & 6 & 45.23 & 3.2 & 6 & $\%$ \\
\hline & Mean corpuscular volume & 63.38 & 0.97 & 6 & 67.18 & 1.55 & 4 & 67.52 & 2.46 & 6 & 67.1 & 1.42 & 6 & $f L$ \\
\hline & Mean corpuscular hemoglobin & 22.1 & 0.3 & 6 & 21.58 & 0.62 & 4 & 22.12 & 0.78 & 6 & 21.95 & 0.76 & & $\mathrm{pg}$ \\
\hline & Mean corpuscular hemoglobin concentration & 32.37 & 0.18 & 6 & 32.13 & 0.61 & 4 & 32.73 & 0.2 & 6 & 32.73 & 0.56 & 6 & $\mathrm{~g} / \mathrm{dL}$ \\
\hline & Reticulocytes & 24.63 & 13.54 & 6 & 29.25 & 7.68 & 4 & 22.28 & 5.7 & 6 & 21.75 & 10 & 6 & $10^{9} / \mathrm{L}$ \\
\hline & Leukocytes & & & & & & & & & & & & & \\
\hline & White blood cells & 7.473 & 1.869 & 6 & 7.373 & 1.858 & 4 & 9.175 & 2.951 & 6 & 7.103 & 1.64 & 6 & K/uL \\
\hline & Neutrophils & 1.207 & 1.602 & 6 & 3.9 & 1.37 & 4 & 5.428 & 2.489 & 6 & 3.943 & 1.249 & 6 & KuL \\
\hline & Lymphocytes & 2.473 & 0.55 & 6 & 2.69 & 0.506 & 4 & 2.638 & 0.788 & 6 & 2.445 & 0.445 & 6 & KuL \\
\hline & Monocytes & 0.512 & 0.275 & 6 & 0.54 & 0.083 & 4 & 0.668 & 0.413 & 6 & 0.375 & 0.121 & 6 & KuL \\
\hline & Eosinophils & 0.128 & 0.077 & 6 & 0.14 & 0.022 & 4 & 0.282 & 0.171 & 6 & 0.193 & 0.108 & 6 & KuL \\
\hline & Basophils & 0.093 & 0.051 & 6 & 0.063 & 0.021 & 4 & 0.09 & 0.029 & 6 & 0.082 & 0.018 & 4 & K/uL \\
\hline & Thrombocytes & & & & & & & & & & & & & \\
\hline & Platelets & 381.7 & 39.4 & 6 & 330.8 & 80.1 & 4 & 427.8 & 82.2 & 6 & 360 & 57.4 & 6 & K/uL \\
\hline & Mean platelet volume & 12.38 & 1.52 & 6 & 14.28 & 2.15 & 4 & 12.62 & 1.17 & 6 & 13.53 & 0.9 & 6 & $f L$ \\
\hline & Protrombin time & 6.63 & 0.24 & 6 & 6.88 & 0.17 & 4 & 6.8 & 0.3 & 6 & 6.85 & 0.19 & 6 & Seconds \\
\hline & Activated partial thromboplastin time & 13.12 & 0.82 & 6 & 12.9 & 0.66 & 4 & 13.83 & 0.81 & 6 & 13.33 & 0.83 & 6 & Seconds \\
\hline & Fibrinogen & 270.2 & 47.3 & 6 & 312.5 & 47.5 & 4 & 282 & 44.6 & 6 & 259.8 & 56.7 & 6 & $\mathrm{Mg} / \mathrm{dL}$ \\
\hline \multirow[t]{21}{*}{ Females } & Erythrocytes & & & & & & & & & & & & & \\
\hline & Red blood cells & 6.562 & 0.322 & 6 & 6.788 & 0.297 & 4 & 6.49 & 0.417 & 6 & 6.59 & 0.344 & 6 & M/ul \\
\hline & Hemoglobin & 14.62 & 0.8 & 6 & 15.43 & 0.77 & 4 & 14.12 & 1.52 & 6 & 14.7 & 0.79 & 6 & $\mathrm{~g} / \mathrm{L}$ \\
\hline & Hematocrit & 45.15 & 2.42 & 6 & 46.98 & 2.33 & 4 & 43.78 & 3.88 & 6 & 45.22 & 2.26 & 6 & $\%$ \\
\hline & Mean corpuscular volume & 68.82 & 2.46 & 6 & 69.2 & 1.83 & 4 & 67.4 & 2.3 & 6 & 68.63 & 1.52 & 6 & $f \mathrm{~L}$ \\
\hline & Mean corpuscular volume hemoglobin & 22.3 & 0.58 & 6 & 22.75 & 0.83 & 4 & 21.73 & 1.12 & 6 & 22.32 & 0.61 & 6 & $\mathrm{Pg}$ \\
\hline & Mean corpuscular hemoglobin concentration & 32.4 & 0.41 & 6 & 32.85 & 0.31 & 4 & 32.17 & 0.65 & 6 & 32.55 & 0.21 & 6 & $\mathrm{~g} / \mathrm{dL}$ \\
\hline & Reticulocytes & 32.4 & 15.63 & 6 & 41.9 & 19.22 & 4 & 34.42 & 14.98 & 6 & 25.3 & 11.84 & 6 & $10^{9} / \mathrm{L}$ \\
\hline & Leukocytes & & & & & & & & & & & & & \\
\hline & White blood cells & 8.123 & 1.412 & 6 & 8.54 & 1.517 & 4 & 8.418 & 2.205 & 6 & 8.655 & 1.246 & 6 & KuL \\
\hline & Neutrophils & 4.873 & 1.151 & 6 & 4.855 & 1.139 & 4 & 4.88 & 1.404 & 6 & 5.457 & 0.986 & 6 & KuL \\
\hline & Lymphocytes & 2.482 & 0.62 & 6 & 2.793 & 0.381 & 4 & 2.578 & 0.459 & 6 & 2.482 & 0.213 & 6 & KuL \\
\hline & Monocytes & 0.473 & 0.079 & 6 & 0.513 & 0.131 & 4 & 0.505 & 0.185 & 6 & 0.353 & 0.119 & 6 & K/uL \\
\hline & Eosinophils & 0.15 & 0.14 & 6 & 0.138 & 0.019 & 4 & 0.313 & 0.324 & 6 & 0.197 & 0.072 & 6 & KuL \\
\hline & Basophils & 0.093 & 0.047 & 6 & 0.148 & 0.038 & 4 & 0.08 & 0.029 & 6 & 0.102 & 0.014 & 6 & K/uL \\
\hline & Thrombocytes & & & & & & & & & & & & & \\
\hline & platelets & 358.8 & 83 & 6 & 350.5 & 81.3 & 4 & 319.2 & 53.1 & 6 & 356.7 & 49.3 & 6 & K/uL \\
\hline & Mean platelet volume & 13.23 & 2.08 & 6 & 13.13 & 1.27 & 4 & 13.43 & 1.24 & 6 & 12.85 & 1.05 & 6 & $f L$ \\
\hline & Prothrombin time & 6.9 & 0.32 & 6 & 7.03 & 0.25 & 4 & 6.73 & 0.36 & 6 & 6.97 & 0.34 & 6 & Seconds \\
\hline & Activated partial thromboplastin time & 13.42 & 0.65 & 6 & 13.4 & 0.41 & 4 & 13.37 & 0.44 & 6 & 13.12 & 0.37 & 6 & Seconds \\
\hline & Fibrinogen & 227 & 54.8 & 6 & 225.8 & 90.2 & 4 & 219.2 & 42.6 & 6 & 205.8 & 35.3 & 6 & $\mathrm{mg} / \mathrm{dL}$ \\
\hline
\end{tabular}


Table 4 Mean blood cells and coagulation parameters in dogs 29 days after the start date

\begin{tabular}{|c|c|c|c|c|c|c|c|c|c|c|c|c|c|c|}
\hline & & \multicolumn{3}{|c|}{ Vehicle } & \multicolumn{3}{|c|}{100 mg/kg } & \multicolumn{3}{|c|}{300 mg/kg } & \multicolumn{3}{|c|}{1500 mg/kg } & \multirow[t]{2}{*}{ Unit } \\
\hline & & Mean & SD & N & Mean & SD & $\mathrm{N}$ & Mean & SD & N & Mean & SD & $N$ & \\
\hline \multirow[t]{21}{*}{ Males } & Erythrocytes & & & & & & & & & & & & & \\
\hline & Red blood cells & 6.238 & 0.841 & 4 & 6.438 & 0.598 & 4 & 6.21 & 0.143 & 4 & 6.418 & 0.437 & 4 & M/ul \\
\hline & Hemoglobin & 14.55 & 1.91 & 4 & 14.55 & 1.1 & 4 & 6.21 & 0.143 & 4 & 6.418 & 0.437 & 4 & $g / L$ \\
\hline & Hematocrit & 42.1 & 6.09 & 4 & 42.18 & 3.27 & 4 & 41.05 & 1.04 & 4 & 41.7 & 2.79 & 4 & $\%$ \\
\hline & Mean corpuscular volume & 67.45 & 1.1 & 4 & 65.63 & 1.51 & 4 & 66.08 & 2.16 & 4 & 65 & 1.75 & 4 & $f L$ \\
\hline & Mean corpuscular hemoglobin & 23.35 & 0.24 & 4 & 22.68 & 0.59 & 4 & 22.9 & 0.64 & 4 & 22.73 & 0.7 & 4 & $\mathrm{pg}$ \\
\hline & Mean corpuscular hemoglobin concentration & 34.6 & 0.47 & 4 & 34.6 & 0.37 & 4 & 34.63 & 0.33 & 4 & 34.9 & 0.51 & 4 & $\mathrm{~g} / \mathrm{dL}$ \\
\hline & Reticulocytes & 42.73 & 11.31 & 4 & 58.65 & 15.92 & 4 & 46 & 11.97 & 4 & 24.55 & 3.67 & 4 & $10^{9} / \mathrm{L}$ \\
\hline & Leukocytes & & & & & & & & & & & & & \\
\hline & White blood cells & 7.438 & 0.919 & 4 & 9.23 & 1.25 & 4 & 12.23 & 3.277 & 4 & 11.91 & 3.574 & 4 & K/uL \\
\hline & Neutrophils & 4.183 & 0.937 & 4 & 4.805 & 0.658 & 4 & 7.438 & 3.162 & 4 & 7.193 & 2.464 & 4 & KuL \\
\hline & Lymphocytes & 2.593 & 0.442 & 4 & 3.438 & 0.597 & 4 & 3.56 & 0.326 & 4 & 3.655 & 0.792 & 4 & KuL \\
\hline & Monocytes & 0.43 & 0.153 & 4 & 0.683 & 0.089 & 4 & 0.825 & 0.26 & 4 & 0.565 & 0.14 & 4 & KuL \\
\hline & Eosinophils & 0.095 & 0.039 & 4 & 0.178 & 0.055 & 4 & 0.223 & 0.101 & 4 & 0.388 & 0.328 & 4 & KuL \\
\hline & Basophils & 0.098 & 0.078 & 4 & 0.1 & 0.018 & 4 & 0.138 & 0.038 & 4 & 0.08 & 0.026 & 4 & K/uL \\
\hline & Thrombocytes & & & & & & & & & & & & & \\
\hline & platelets & 259.8 & 64 & 4 & 281.8 & 44.7 & 4 & 369.5 & 87.3 & 4 & 262.3 & 75.2 & 4 & K/uL \\
\hline & Mean platelet volume & 10.95 & 1.11 & 4 & 9.88 & 0.8 & 4 & 9 & 0.87 & 4 & 8.6 & 0.77 & 4 & $f L$ \\
\hline & Protrombin time & 6.78 & 0.05 & 4 & 7.08 & 0.19 & 4 & 6.85 & 0.17 & 4 & 6.9 & 0.24 & 4 & Seconds \\
\hline & Activated partial thromboplastin time & 12.68 & 1.16 & 4 & 12.7 & 0.84 & 4 & 13.5 & 0.88 & 4 & 13.05 & 1.28 & 4 & Seconds \\
\hline & Fibrinogen & 254.5 & 42.7 & 4 & 288.5 & 18.9 & 4 & 289.5 & 85.4 & 4 & 414.5 & 89.9 & 4 & $\mathrm{mg} / \mathrm{dL}$ \\
\hline \multirow[t]{21}{*}{ Females } & Erythrocytes & & & & & & & & & & & & & \\
\hline & Red blood cells & 6.33 & 0.241 & 4 & 6.895 & 0.563 & 4 & 6.72 & 0.306 & 4 & 6.773 & 0.383 & 4 & M/ul \\
\hline & Hemoglobin & 14.85 & 1 & 4 & 16.43 & 1.7 & 4 & 15.55 & 1.1 & 4 & 15.78 & 0.8 & 4 & $\mathrm{~g} / \mathrm{L}$ \\
\hline & Hematocrit & 43.33 & 3.14 & 4 & 47.68 & 4.73 & 4 & 45.48 & 3.24 & 4 & 45.98 & 2.05 & 4 & $\%$ \\
\hline & Mean corpuscular volume & 68.33 & 2.53 & 4 & 69.1 & 1.14 & 4 & 67.83 & 3.07 & 4 & 67.88 & 1.6 & 4 & $f \mathrm{~L}$ \\
\hline & Mean corpuscular volume hemoglobin & 23.45 & 0.71 & 4 & 23.83 & 0.55 & 4 & 23.15 & 1 & 4 & 23.3 & 0.55 & 4 & $\mathrm{pg}$ \\
\hline & Mean corpuscular hemoglobin concentration & 34.25 & 0.47 & 4 & 69.3 & 23.65 & 4 & 72.85 & 24.88 & 4 & 45.75 & 23.65 & 4 & $\mathrm{~g} / \mathrm{dL}$ \\
\hline & Reticulocytes & 34.25 & 0.47 & 4 & 34.5 & 0.24 & 4 & 34.23 & 0.35 & 4 & 34.3 & 0.46 & 4 & $10^{9} / \mathrm{L}$ \\
\hline & Leukocytes & & & & & & & & & & & & & \\
\hline & White blood cells & 8.81 & 3.586 & 4 & 9.75 & 2.4 & 4 & 9.743 & 1.237 & 4 & 13.31 & 1.721 & 4 & KuL \\
\hline & Neutrophils & 5.293 & 3.009 & 4 & 4.928 & 1.273 & 4 & 5.32 & 1.312 & 4 & 8.193 & 1.654 & 4 & KuL \\
\hline & Lymphocytes & 2.693 & 0.318 & 4 & 3.575 & 0.702 & 4 & 3.358 & 0.543 & 4 & 3.985 & 0.625 & 4 & KuL \\
\hline & Monocytes & 0.548 & 0.218 & 4 & 0.495 & 0.071 & 4 & 0.648 & 0.083 & 4 & 0.665 & 0.281 & 4 & K/uL \\
\hline & Eosinophils & 0.163 & 0.111 & 4 & 0.505 & 0.467 & 4 & 0.273 & 0.1 & 4 & 0.3 & 0.124 & 4 & KuL \\
\hline & Basophils & 0.078 & 0.022 & 4 & 0.175 & 0.027 & 4 & 0.105 & 0.019 & 4 & 0.123 & 0.051 & 4 & K/uL \\
\hline & Thrombocytes & & & & & & & & & & & & & \\
\hline & platelets & 275.8 & 62.7 & 4 & 301.3 & 31.9 & 4 & 294.3 & 79 & 4 & 340 & 72.2 & 4 & K/uL \\
\hline & Mean platelet volume & 13.03 & 2.23 & 4 & 10.45 & 0.79 & 4 & 10.85 & 0.97 & 4 & 9.75 & 0.71 & 4 & $f L$ \\
\hline & Prothrombin time & 7.1 & 0.18 & 4 & 7.18 & 0.15 & 4 & 6.8 & 0.22 & 4 & 6.93 & 0.19 & 4 & Seconds \\
\hline & Activated partial thromboplastin time & 12.23 & 0.72 & 4 & 12.68 & 0.92 & 4 & 13.48 & 0.4 & 4 & 12.45 & 0.47 & 4 & Seconds \\
\hline & Fibrinogen & 206 & 11.1 & 4 & 178.5 & 16.3 & 4 & 251.5 & 68.3 & 4 & 275.8 & 58.8 & 4 & $\mathrm{mg} / \mathrm{dL}$ \\
\hline
\end{tabular}


Table 5 Mean blood cells and coagulation parameters in dogs at the end of the recovery period (Day 57)

\begin{tabular}{|c|c|c|c|c|c|c|c|c|}
\hline & & \multicolumn{2}{|c|}{ Vehicle } & \multicolumn{2}{|c|}{$300 \mathrm{mg} / \mathrm{kg}$} & \multicolumn{2}{|c|}{$1500 \mathrm{mg} / \mathrm{kg}$} & \multirow[t]{2}{*}{ Unit } \\
\hline & & $\overline{M e a n}$ & $\bar{N}$ & $\overline{\text { Mean }}$ & $\mathrm{N}$ & $\overline{\text { Mean }}$ & $\mathrm{N}$ & \\
\hline \multirow[t]{21}{*}{ Males } & Erythrocytes & & & & & & & \\
\hline & Red blood cells & 5.975 & 2 & 6.13 & 2 & 6.115 & 2 & $\mathrm{M} / \mathrm{ul}$ \\
\hline & Hemoglobin & 14 & 2 & 14.2 & 2 & 14.15 & 2 & $g / L$ \\
\hline & Hematocrit & 40 & 2 & 40.7 & 2 & 40.65 & 2 & $\%$ \\
\hline & Mean corpuscular volume & 67 & 2 & 6.35 & 2 & 66.7 & 2 & $\mathrm{fL}$ \\
\hline & Mean corpuscular hemoglobin & 23.4 & 2 & 23.15 & 2 & 23.2 & 2 & $\mathrm{pg}$ \\
\hline & Mean corpuscular hemoglobin concentration & 34.9 & 2 & 34.85 & 2 & 34.8 & 2 & $\mathrm{~g} / \mathrm{dL}$ \\
\hline & Reticulocytes & 34.15 & 2 & 78.3 & 2 & 59.92 & 2 & $10^{9} / \mathrm{L}$ \\
\hline & Leukocytes & & & & & & & \\
\hline & White blood cells & 8.705 & 2 & 8.025 & 2 & 10.37 & 2 & K/uL \\
\hline & Neutrophils & 5.275 & 2 & 4.53 & 2 & 6.14 & 2 & KuL \\
\hline & Lymphocytes & 2.7 & 2 & 2.645 & 2 & 3.055 & 2 & KuL \\
\hline & Monocytes & 0.34 & 2 & 0.225 & 2 & 0.385 & 2 & K/uL \\
\hline & Eosinophils & 0.21 & 2 & 0.225 & 2 & 0.385 & 2 & KuL \\
\hline & Basophils & 0.125 & 2 & 0.125 & 2 & 0.125 & 2 & KuL \\
\hline & Thrombocytes & & & & & & & \\
\hline & platelets & 229 & 2 & 290.5 & 2 & 310 & 2 & KuL \\
\hline & Mean platelet volume & 11.7 & 2 & 9.65 & 2 & 9.55 & 2 & $\mathrm{fL}$ \\
\hline & Protrombin time & 7.05 & 2 & 7.15 & 2 & 7.15 & 2 & Seconds \\
\hline & Activated partial thromboplastin time & 13.05 & 2 & 13.75 & 2 & 12.65 & 2 & Seconds \\
\hline & Fibrinogen & & & & & & & $\mathrm{mg} / \mathrm{dL}$ \\
\hline \multirow[t]{21}{*}{ Females } & Erythrocytes & & & & & & & \\
\hline & Red blood cells & 7.41 & 2 & 6.775 & 2 & 6.455 & 2 & $\mathrm{M} / \mathrm{ul}$ \\
\hline & Hemoglobin & 17.25 & 2 & 15.7 & 2 & 15.05 & 2 & $g / L$ \\
\hline & Hematocrit & 49.65 & 2 & 44.75 & 2 & 43.25 & 2 & $\%$ \\
\hline & Mean corpuscular volume & 66.95 & 2 & 66 & 2 & 66.8 & 2 & $\mathrm{fL}$ \\
\hline & Mean corpuscular volume hemoglobin & 23.3 & 2 & 23.15 & 2 & 23.3 & 2 & $\mathrm{pg}$ \\
\hline & Mean corpuscular hemoglobin concentration & 34.8 & 2 & 35.1 & 2 & 34.85 & 2 & $\mathrm{~g} / \mathrm{dL}$ \\
\hline & Reticulocytes & 69.4 & 2 & 68.25 & 2 & 52.3 & 2 & $10^{9} / \mathrm{L}$ \\
\hline & Leukocytes & & & & & & & \\
\hline & White blood cells & 8.79 & 2 & 7.2 & 2 & 8.01 & 2 & KuL \\
\hline & Neutrophils & 4.925 & 2 & 3.895 & 2 & 4.915 & 2 & KuL \\
\hline & Lymphocytes & 2.87 & 2 & 2.785 & 2 & 2.475 & 2 & KuL \\
\hline & Monocytes & 0.34 & 2 & 0.215 & 2 & 0.275 & 2 & KuL \\
\hline & Eosinophils & 0445 & 2 & 0.16 & 2 & 0.2 & 2 & K/uL \\
\hline & Basophils & 0.165 & 2 & 0.11 & 2 & 0.11 & 2 & KuL \\
\hline & Thrombocytes & & & & & & & \\
\hline & platelets & 239.5 & 2 & 273 & 2 & 274.5 & 2 & KuL \\
\hline & Mean platelet volume & 11.3 & 2 & 11.2 & 2 & 10 & 2 & $\mathrm{fL}$ \\
\hline & Prothrombin time & 6.7 & 2 & 7.35 & 2 & 6.8 & 2 & Seconds \\
\hline & Activated partial thromboplastin time & 12.6 & 2 & 12.3 & 2 & 12.7 & 2 & Seconds \\
\hline & Fibrinogen & 291 & 2 & 151.5 & 2 & 201.5 & 2 & $\mathrm{mg} / \mathrm{dL}$ \\
\hline
\end{tabular}


Table 6 Mean serum chemistry parameters in dogs 10 days prior to treatment

\begin{tabular}{|c|c|c|c|c|c|c|c|c|c|c|c|c|c|c|}
\hline & & \multicolumn{3}{|c|}{ Vehicle } & \multicolumn{3}{|c|}{$100 \mathrm{mg} / \mathrm{kg}$} & \multicolumn{3}{|c|}{300 mg/kg } & \multicolumn{3}{|c|}{1500 mg/kg } & \multirow[t]{2}{*}{ Unit } \\
\hline & & Mean & SD & $\mathrm{N}$ & Mean & SD & N & Mean & SD & $\mathrm{N}$ & Mean & SD & $\mathrm{N}$ & \\
\hline \multirow[t]{21}{*}{ Males } & Alanine transaminase (ALT) & 58.2 & 47.5 & 6 & 55.8 & 42.2 & 4 & 37.7 & 5.4 & 6 & 36.8 & 15.2 & 6 & $U / L$ \\
\hline & Aspartate transaminase (AST) & 27.3 & 2.1 & 6 & 29.8 & 5.4 & 4 & 32.5 & 3.8 & 6 & 29.7 & 3.7 & 8 & $U / L$ \\
\hline & Alkaline phosphatase (ALKP) & 91.2 & 20.5 & 6 & 109.8 & 39.9 & 4 & 93.8 & 21.7 & 6 & 128.7 & 40.1 & 6 & $\mathrm{U} / \mathrm{L}$ \\
\hline & Lactate deshydrogenase (LDH) & 75.3 & 24.7 & 6 & 71.3 & 14.4 & 4 & 76 & 13.8 & 6 & 67.5 & 24.5 & 6 & $\mathrm{U} / \mathrm{L}$ \\
\hline & Total bilirubin (TBILI) & 0 & 0 & 6 & 0 & 0 & 4 & 0 & 0 & 0 & 0 & 0 & 6 & $\mathrm{mg} / \mathrm{dL}$ \\
\hline & Gamma-Glutamyl transferase (GGT) & 0 & 0 & 6 & 0 & 0 & 4 & 0 & 0 & 6 & 0 & 0 & 6 & $\mathrm{U} / \mathrm{L}$ \\
\hline & Creatine phosphokinase (CK) & 0 & 0 & 6 & 0 & 0 & 4 & 0 & 0 & 4 & 0 & 0 & 6 & $\mathrm{U} / \mathrm{L}$ \\
\hline & Sodium (Na) & 146 & 1.5 & 6 & 145.5 & 1 & 4 & 146.2 & 1.3 & 6 & 146.7 & 0.8 & 6 & $\mathrm{mmol} / \mathrm{L}$ \\
\hline & Potassium (K) & 4.65 & 0.19 & 6 & 4.65 & 0.17 & 4 & 113.3 & 1 & 6 & 113 & 1.1 & 6 & $\mathrm{mmol} / \mathrm{L}$ \\
\hline & Chloride (CL) & 113.5 & 1.5 & 6 & 113.3 & 1 & 4 & 113 & 1.1 & 6 & 113.5 & 2.4 & 6 & $\mathrm{mmol} / \mathrm{L}$ \\
\hline & Calcium (CA) & 10.13 & 0.19 & 6 & 10.28 & 0.15 & 4 & 10.43 & 0.3 & 6 & 10.53 & 0.45 & 0 & $\mathrm{mg} / \mathrm{dL}$ \\
\hline & Inorganic phosphorus (PHOS) & 5.43 & 0.77 & 6 & 5.98 & 0.7 & 4 & 5.23 & 0.38 & 6 & 5.3 & 0.45 & 6 & $\mathrm{mg} / \mathrm{dL}$ \\
\hline & Blood urea nitrogen (BUN) & 14.2 & 0.8 & 6 & 14.5 & 0.6 & 4 & 17 & 2.7 & 6 & 16 & 2.1 & 6 & $\mathrm{mg} / \mathrm{dL}$ \\
\hline & Creatinine (CREA) & 0.63 & 0.12 & 6 & 0.58 & 0.05 & 4 & 0.6 & 0 & 6 & 0.67 & 0.08 & 6 & $\mathrm{mg} / \mathrm{dL}$ \\
\hline & Glucose (GLU) & 97.3 & 3.5 & 5 & 91.3 & 8.3 & 4 & 92.3 & 7.4 & 6 & 96 & 8.9 & 6 & $\mathrm{mg} / \mathrm{dL}$ \\
\hline & Total protein (TPRO) & 4.75 & 0.24 & 6 & 4.98 & 0.29 & 4 & 4.9 & 0.14 & 6 & 4.87 & 0.26 & 6 & $\mathrm{~g} / \mathrm{dL}$ \\
\hline & Albumin (ALB) & 2.88 & 0.17 & 6 & 2.93 & 0.26 & 4 & 2.92 & 0.12 & 6 & 2.83 & 0.28 & 6 & $\mathrm{~g} / \mathrm{dL}$ \\
\hline & Globulin (GLOB) & 1.87 & 0.18 & 6 & 2.05 & 0.13 & 4 & 1.98 & 0.04 & 6 & 2.03 & 0.23 & 6 & $\mathrm{~g} / \mathrm{dL}$ \\
\hline & Albumin/Globulin ratio (A/G) & 1.555 & 0.165 & 6 & 1.431 & 0.155 & 4 & 1.471 & 0.051 & 6 & 1.415 & 0.249 & 6 & Ratio \\
\hline & Cholesterol (CHOL) & 168.3 & 48.5 & 6 & 160.8 & 9.3 & 4 & 150.5 & 31.5 & 6 & 163.8 & 24 & 6 & $\mathrm{mg} / \mathrm{dL}$ \\
\hline & Triglycerides (TRIG) & 37.3 & 8.2 & 6 & 38.3 & 5.9 & 4 & 36 & 4 & 6 & 40.2 & 5 & 6 & $\mathrm{mg} / \mathrm{dL}$ \\
\hline \multirow[t]{21}{*}{ Females } & Alanine transaminase (ALT) & 31.8 & 6.6 & 6 & 32.8 & 7.4 & 4 & 30.3 & 4.1 & 6 & 37.5 & 16.3 & 6 & U/L \\
\hline & Aspartate transaminase (AST) & 29.3 & 4.1 & 6 & 31.8 & 11.2 & 4 & 27.5 & 3.4 & 6 & 28.3 & 3.7 & 6 & $U / L$ \\
\hline & Alkaline phosphatase (ALKP) & 100 & 32 & 6 & 83.8 & 18.3 & 4 & 95.7 & 25.5 & 6 & 75.3 & 13.7 & 6 & $U / L$ \\
\hline & Lactate deshydrogenase (LDH) & 60.3 & 18.1 & 6 & 65.3 & 25.1 & 4 & 52.2 & 15.4 & 6 & 62.2 & 21.8 & 6 & $U / L$ \\
\hline & Total bilirubin (TBILI) & 0.02 & 0.04 & 6 & 0 & 0 & 4 & 0 & 0 & 6 & 0 & 0 & 6 & $\mathrm{mg} / \mathrm{dL}$ \\
\hline & Gamma-Glutamyl transferase (GGT) & 0 & 0 & 6 & 0 & 0 & 4 & 0 & 0 & 6 & 0 & 0 & 6 & $U / L$ \\
\hline & Creatine phosphokinase (CK) & 165.7 & 37.4 & 6 & 161.5 & 73.7 & 4 & 141 & 30.1 & 6 & 142 & 43.6 & 6 & $U / L$ \\
\hline & Sodium (Na) & 146.8 & 1.5 & 6 & 147.3 & 1.7 & 4 & 146.7 & 1 & 6 & 147.3 & 0.8 & 6 & $\mathrm{mmol} / \mathrm{L}$ \\
\hline & Potassium (K) & 4.48 & 0.26 & 6 & 4.48 & 0.05 & 4 & 4.43 & 0.31 & 6 & 4.43 & 0.21 & 6 & $\mathrm{mmol} / \mathrm{L}$ \\
\hline & Chloride (CL) & 114.2 & 2.1 & 6 & 116 & 2.2 & 4 & 114.7 & 2.9 & 6 & 115 & 1.3 & 6 & $\mathrm{mmol} / \mathrm{L}$ \\
\hline & Calcium (CA) & 10.48 & 0.26 & 6 & 10.58 & 0.21 & 4 & 10.95 & 0.27 & 6 & 10.82 & 0.31 & 6 & $\mathrm{mg} / \mathrm{dL}$ \\
\hline & Inorganic phosphorus (PHOS) & 5.3 & 0.23 & 6 & 4.83 & 0.31 & 4 & 5.35 & 0.55 & 6 & 5.17 & 0.5 & 6 & $\mathrm{mg} / \mathrm{dL}$ \\
\hline & Blood urea nitrogen (BUN) & 15.2 & 2.3 & 6 & 13.3 & 1 & 4 & 14.2 & 2.6 & 6 & 14 & 3 & 6 & $\mathrm{mg} / \mathrm{dL}$ \\
\hline & Creatinine (CREA) & 0.57 & 0.05 & 6 & 0.6 & 0 & 4 & 0.58 & 0.08 & 6 & 0.65 & 0.08 & 6 & $\mathrm{mg} / \mathrm{dL}$ \\
\hline & Glucose (GLU) & 99.3 & 5 & 6 & 99.3 & 7.5 & 4 & 99.7 & 7.4 & 6 & 94.5 & 7 & 6 & $\mathrm{mg} / \mathrm{dL}$ \\
\hline & Total protein (TPRO) & 4.85 & 0.27 & 6 & 5 & 0.28 & 4 & 4.8 & 0.14 & 6 & 5.13 & 0.16 & 6 & $\mathrm{~g} / \mathrm{dL}$ \\
\hline & Albumin (ALB) & 2.95 & 0.26 & 6 & 3.1 & 0.14 & 4 & 2.95 & 0.18 & 6 & 3.13 & 0.16 & 4 & $\mathrm{~g} / \mathrm{dL}$ \\
\hline & Globulin (GLOB) & 1.9 & 0.09 & 6 & 1.9 & 0.18 & 4 & 1.85 & 0.14 & 6 & 2 & 0 & 6 & $\mathrm{~g} / \mathrm{dL}$ \\
\hline & Albumin/Globulin ratio (A/G) & 1.556 & 0.157 & 6 & 162.3 & 10.5 & 4 & 1.607 & 0.21 & 6 & 1.567 & 0.082 & 6 & Ratio \\
\hline & Cholesterol (CHOL) & 160 & 20.2 & 6 & 162.3 & 10.5 & 4 & 142.3 & 11.3 & 6 & 158.2 & 33 & 6 & $\mathrm{mg} / \mathrm{dL}$ \\
\hline & Triglycerides (TRIG) & 37.3 & 3.8 & 6 & 36 & 14.1 & 4 & 34 & 4.8 & 6 & 46.3 & 10.4 & 6 & $\mathrm{mg} / \mathrm{dL}$ \\
\hline
\end{tabular}


Table 7 Mean serum chemistry parameters 29 days after the start date in male dogs

\begin{tabular}{|c|c|c|c|c|c|c|c|c|c|c|c|c|c|c|}
\hline & & \multicolumn{3}{|c|}{ Vehicle } & \multicolumn{3}{|c|}{100 mg/kg } & \multicolumn{3}{|c|}{300 mg/kg } & \multicolumn{3}{|c|}{1500 mg/kg } & \multirow[t]{2}{*}{ Unit } \\
\hline & & Mean & SD & $\bar{N}$ & Mean & SD & $\bar{N}$ & Mean & SD & $\bar{N}$ & Mean & SD & $\bar{N}$ & \\
\hline \multirow[t]{21}{*}{ Males } & Alanine transaminase (ALT) & 33.5 & 9.9 & 4 & 32 & 8.6 & 4 & 37.3 & 6.8 & 4 & 51 & 25.9 & 4 & $U / L$ \\
\hline & Aspartate transaminase (AST) & 34.8 & 3.2 & 4 & 36.5 & 5.7 & 4 & 46 & 8.8 & 4 & 49.8 & 5.9 & 4 & $U / L$ \\
\hline & Alkaline phosphatase (ALKP) & 96 & 24.4 & 4 & 104.3 & 37.1 & 4 & 116 & 71.9 & 4 & 227 & 315.9 & 4 & $\mathrm{U} / \mathrm{L}$ \\
\hline & Lactate deshydrogenase (LDH) & 57.8 & 6.4 & 4 & 61 & 18.7 & 4 & 70 & 21.8 & 4 & 55 & 18.9 & 4 & $\mathrm{U} / \mathrm{L}$ \\
\hline & Total bilirubin (TBILI) & 0.03 & 0.05 & 4 & 0.03 & 0.05 & 4 & 0.03 & 0.05 & 4 & 0 & 0 & 4 & $\mathrm{mg} / \mathrm{dL}$ \\
\hline & Gamma-Glutamyl transferase (GGT) & 0 & 0 & 4 & 0 & 0 & 4 & 0 & 0 & 4 & 0 & 0 & 4 & $U / L$ \\
\hline & Creatine phosphokinase (CK) & 246.3 & 79.8 & 4 & 185.3 & 61.2 & 4 & 272.3 & 145.6 & 4 & 133 & 29.1 & 4 & $U / L$ \\
\hline & Sodium (Na) & 148.5 & 1.3 & 4 & 148 & 0.8 & 4 & 147.8 & 0.5 & 4 & 148 & 1.4 & 4 & $\mathrm{mmol} / \mathrm{L}$ \\
\hline & Potassium (K) & 4.35 & 0.31 & 4 & 4.5 & 0.26 & 4 & 4.08 & 0.32 & 4 & 4.48 & 0.21 & 4 & $\mathrm{mmol} / \mathrm{L}$ \\
\hline & Chloride (CL) & 115.8 & 1 & 4 & 115 & 0.8 & 4 & 115 & 1.3 & 4 & 118.8 & 0.5 & 4 & $\mathrm{mmol} / \mathrm{L}$ \\
\hline & Calcium (CA) & 10.83 & 0.25 & 4 & 10.88 & 0.33 & 4 & 11.05 & 0.13 & 4 & 10.35 & 0.29 & 4 & $\mathrm{mg} / \mathrm{dL}$ \\
\hline & Inorganic phosphorus (PHOS) & 5.13 & 0.75 & 4 & 5.45 & 0.29 & 4 & 4.68 & 0.26 & 4 & 3.98 & 0.32 & 4 & $\mathrm{mg} / \mathrm{dL}$ \\
\hline & Blood urea nitrogen (BUN) & 18.3 & 2.1 & 4 & 18.8 & 3.3 & 4 & 19.3 & 1.9 & 4 & 24.3 & 1.7 & 4 & $\mathrm{mg} / \mathrm{dL}$ \\
\hline & Creatinine (CREA) & 0.6 & 0.14 & 4 & 0.6 & 0 & 4 & 0.58 & 0.1 & 4 & 0.55 & 0.08 & 4 & $\mathrm{mg} / \mathrm{dL}$ \\
\hline & Glucose (GLU) & 102.5 & 4 & 4 & 96.3 & 9 & 4 & 96.8 & 11.2 & 4 & 80.8 & 8.4 & 4 & $\mathrm{mg} / \mathrm{dL}$ \\
\hline & Total protein (TPRO) & 5.25 & 0.3 & 4 & 5.38 & 0.28 & 4 & 5.38 & 0.24 & 4 & 4.45 & 0.42 & 4 & $\mathrm{~g} / \mathrm{dL}$ \\
\hline & Albumin (ALB) & 3.33 & 0.26 & 4 & 3.23 & 0.29 & 4 & 3.18 & 0.21 & 4 & 2.45 & 0.1 & 4 & $\mathrm{~g} / \mathrm{dL}$ \\
\hline & Globulin (GLOB) & 1.93 & 0.1 & 4 & 2.15 & 0.06 & 4 & 2.2 & 0.14 & 4 & 2 & 0.34 & 4 & $\mathrm{~g} / \mathrm{dL}$ \\
\hline & Albumin/Globulin ratio (A/G) & 1.729 & 0.141 & 4 & 1.502 & 0.152 & 4 & 1.448 & 0.14 & 4 & 1.247 & 0.184 & 4 & Ratio \\
\hline & Cholesterol (CHOL) & 168.8 & 35.4 & 4 & 176 & 28 & 4 & 152.3 & 19.4 & 4 & 104.5 & 183.4 & 4 & $\mathrm{mg} / \mathrm{dL}$ \\
\hline & Triglycerides (TRIG) & 34 & 8.1 & 4 & 41.3 & 9.7 & 4 & 33.3 & 5.6 & 4 & 45.5 & 14.3 & 4 & $\mathrm{mg} / \mathrm{dL}$ \\
\hline \multirow[t]{21}{*}{ Females } & Alanine transaminase (ALT) & 27.3 & 5.1 & 4 & 26.3 & 1.9 & 4 & 35.8 & 10.2 & 4 & 45.5 & 20.9 & 4 & U/L \\
\hline & Aspartate transaminase (AST) & 29 & 4.7 & 4 & 36.3 & 5.3 & 4 & 31.5 & 3.1 & 4 & 45.8 & 19.5 & 4 & $U / L$ \\
\hline & Alkaline phosphatase (ALKP) & 98.8 & 12.4 & 4 & 78.8 & 22.5 & 4 & 178 & 131.5 & 4 & 251.3 & 211.2 & 4 & $U / L$ \\
\hline & Lactate deshydrogenase (LDH) & 82.3 & 25.8 & 4 & 74 & 19.8 & 4 & 69.5 & 5.9 & 4 & 73 & 19.5 & 4 & $U / L$ \\
\hline & Total bilirubin (TBILI) & 0.03 & 0.05 & 4 & 0.05 & 0.06 & 4 & 0.03 & 0.05 & 4 & 0.03 & 0.05 & 4 & $\mathrm{mg} / \mathrm{dL}$ \\
\hline & Gamma-Glutamyl transferase (GGT) & 0 & 0 & 4 & 0.3 & 0.5 & 4 & 0 & 0 & 4 & 0 & 0 & 4 & $U / L$ \\
\hline & Creatine phosphokinase (CK) & 162.8 & 54.3 & 4 & 198.8 & 44.1 & 4 & 162.3 & 40.6 & 4 & 147.3 & 47.6 & 4 & $U / L$ \\
\hline & Sodium (Na) & 147.8 & 1 & 4 & 148.5 & 1.9 & 4 & 148.5 & 0.6 & 4 & 148.3 & 1 & 4 & $\mathrm{mmol} / \mathrm{L}$ \\
\hline & Potassium (K) & 4.4 & 0.34 & 4 & 4.53 & 0.17 & 4 & 4.38 & 0.31 & 4 & 4.65 & 0.34 & 4 & $\mathrm{mmol} / \mathrm{L}$ \\
\hline & Chloride (CL) & 114.5 & 1.7 & 4 & 115.5 & 1 & 4 & 115.5 & 1 & 4 & 117.8 & 2.8 & 4 & $\mathrm{mmol} / \mathrm{L}$ \\
\hline & Calcium (CA) & 10.7 & 0.35 & 4 & 11.05 & 0.38 & 4 & 11.05 & 0.37 & 4 & 10.25 & 0.84 & 4 & $\mathrm{mg} / \mathrm{dL}$ \\
\hline & Inorganic phosphorus (PHOS) & 5.03 & 0.54 & 4 & 4.25 & 0.9 & 4 & 4.4 & 0.47 & 4 & 4.35 & 0.58 & 4 & $\mathrm{mg} / \mathrm{dL}$ \\
\hline & Blood urea nitrogen (BUN) & 17.5 & 2.4 & 4 & 14.5 & 1.3 & 4 & 18.5 & 1.3 & 4 & 23.5 & 7.9 & 4 & $\mathrm{mg} / \mathrm{dL}$ \\
\hline & Creatinine (CREA) & 0.5 & 0.08 & 4 & 0.58 & 0.1 & 4 & 0.53 & 0.1 & 4 & 0.63 & 0.05 & 4 & $\mathrm{mg} / \mathrm{dL}$ \\
\hline & Glucose (GLU) & 103.3 & 4.8 & 4 & 102.8 & 11.5 & 4 & 108.8 & 7.8 & 4 & 84.8 & 2.1 & 4 & $\mathrm{mg} / \mathrm{dL}$ \\
\hline & Total protein (TPRO) & 5.28 & 0.17 & 4 & 5.35 & 0.31 & 4 & 5.3 & 0.22 & 4 & 4.65 & 0.3 & 4 & $\mathrm{~g} / \mathrm{dL}$ \\
\hline & Albumin (ALB) & 3.35 & 0.17 & 4 & 3.38 & 0.25 & 4 & 3.35 & 0.19 & 4 & 2.8 & 0.22 & 4 & $\mathrm{~g} / \mathrm{dL}$ \\
\hline & Globulin (GLOB) & 1.93 & 0.1 & 4 & 1.98 & 0.1 & 4 & 1.95 & 0.17 & 4 & 1.85 & 0.13 & 4 & $\mathrm{~g} / \mathrm{dL}$ \\
\hline & Albumin/Globulin ration (A/G) & 1.745 & 0.142 & 4 & 1.71 & 0.109 & 4 & 1.729 & 0.191 & 4 & 1.516 & 0.118 & 4 & Ratio \\
\hline & Cholesterol (CHOL) & 167.5 & 24.6 & 4 & 179 & 22.4 & 4 & 147.3 & 10.6 & 4 & 98.3 & 13 & 4 & $\mathrm{mg} / \mathrm{dL}$ \\
\hline & Triglycerides (TRIG) & 40 & 9.8 & 4 & 42 & 8.8 & 4 & 43.5 & 5.8 & 4 & 50 & 8.3 & 4 & $\mathrm{mg} / \mathrm{dL}$ \\
\hline
\end{tabular}


Table 8 Mean serum chemistry parameters in dogs at the end of the recovery period (Day 57)

\begin{tabular}{|c|c|c|c|c|c|c|c|c|}
\hline & & \multicolumn{2}{|c|}{ Vehicle } & \multicolumn{2}{|c|}{300 mg/kg } & \multicolumn{2}{|c|}{1500 mg/kg } & \multirow[t]{2}{*}{ Unit } \\
\hline & & Mean & $\mathrm{N}$ & Mean & $\mathrm{N}$ & Mean & $\mathrm{N}$ & \\
\hline \multirow[t]{21}{*}{ Males } & Alanine transaminase (ALT) & 33 & 2 & 36.5 & 2 & 33 & 2 & $\mathrm{U} / \mathrm{L}$ \\
\hline & Aspartate transaminase (AST) & 31 & 2 & 30.5 & 2 & 33 & 2 & $U / L$ \\
\hline & Alkaline phosphatase (ALKP) & 81.5 & 2 & 66 & 2 & 95 & 2 & $\mathrm{U} / \mathrm{L}$ \\
\hline & Lactate deshydrogenase (LDH) & 36 & 2 & 50 & 2 & 45.5 & 2 & $\mathrm{U} / \mathrm{L}$ \\
\hline & Total bilirubin (TBILI) & 0.05 & 2 & 0.05 & 2 & 0.1 & 2 & $\mathrm{mg} / \mathrm{dl}$ \\
\hline & Gamma-Glutamyl transferase (GGT) & 0 & 2 & 1 & 2 & 0 & 2 & $\mathrm{U} / \mathrm{L}$ \\
\hline & Creatine phosphokinase (CK) & 119 & 2 & 142.5 & 2 & 169 & 2 & $\mathrm{U} / \mathrm{L}$ \\
\hline & Sodium (Na) & 147.5 & 2 & 146.5 & 2 & 148.5 & 2 & $\mathrm{mmol} / \mathrm{L}$ \\
\hline & Potassium (K) & 4.3 & 2 & 4.6 & 2 & 4.4 & 2 & $\mathrm{mmol} / \mathrm{L}$ \\
\hline & Chloride (CL) & 116 & 2 & 115 & 2 & 116.5 & 2 & $\mathrm{mmol} / \mathrm{L}$ \\
\hline & Calcium (CA) & 10.65 & 2 & 10.45 & 2 & 10.55 & 2 & $\mathrm{mg} / \mathrm{dL}$ \\
\hline & Inorganic phosphorus (PHOS) & 5.15 & 2 & 5.1 & 2 & 5.3 & 2 & $\mathrm{mg} / \mathrm{dL}$ \\
\hline & Blood urea nitrogen (BUN) & 13.5 & 2 & 15.5 & 2 & 21 & 2 & $\mathrm{mg} / \mathrm{dL}$ \\
\hline & Creatinine (CREA) & 0.65 & 2 & 0.6 & 2 & 0.7 & 2 & $\mathrm{mg} / \mathrm{dL}$ \\
\hline & Glucose (GLU) & 99 & 2 & 99.5 & 2 & 96 & 2 & $\mathrm{mg} / \mathrm{dL}$ \\
\hline & Total protein (TPRO) & 5.3 & 2 & 5.15 & 2 & 5.4 & 2 & $\mathrm{~g} / \mathrm{dL}$ \\
\hline & Albumin (ALB) & 3 & 2 & 2.9 & 2 & 2.95 & 2 & $\mathrm{~g} / \mathrm{dL}$ \\
\hline & Globulin (GLOB) & 2.3 & 2 & 2.25 & 2 & 2.45 & 2 & $\mathrm{~g} / \mathrm{dL}$ \\
\hline & Albumin/Globulin ratio (A/G) & 1.314 & 2 & 1.289 & 2 & 1.219 & 2 & Ratio \\
\hline & Cholesterol (CHOL) & 149 & 2 & 154 & 2 & 163.5 & 2 & $\mathrm{mg} / \mathrm{dL}$ \\
\hline & Triglycerides (TRIG) & 24 & 2 & 24 & 2 & 41 & 2 & $\mathrm{mg} / \mathrm{dL}$ \\
\hline \multirow[t]{21}{*}{ Females } & Alanine transaminase (ALT) & 22.5 & 2 & 31 & 2 & 27.5 & 2 & $U / L$ \\
\hline & Aspartate transaminase (AST) & 33 & 2 & 29 & 2 & 26.5 & 2 & $U / L$ \\
\hline & Alkaline phosphatase (ALKP) & 73 & 2 & 63.5 & 2 & 50 & 2 & $U / L$ \\
\hline & Lactate deshydrogenase (LDH) & 52 & 2 & 49.5 & 2 & 47 & 2 & $\mathrm{U} / \mathrm{L}$ \\
\hline & Total bilirubin (TBILI) & 0.1 & 2 & 0.1 & 2 & 0.1 & 2 & $\mathrm{mg} / \mathrm{dL}$ \\
\hline & Gamma-Glutamyl transferase (GGT) & 0 & 2 & 0 & 2 & 0 & 0 & $U / L$ \\
\hline & Creatine phosphokinase (CK) & 154.5 & 2 & 146 & 2 & 120 & 2 & $\mathrm{U} / \mathrm{L}$ \\
\hline & Sodium (Na) & 146.5 & 2 & 146.5 & 2 & 147 & 2 & $\mathrm{mmol} / \mathrm{L}$ \\
\hline & Potassium (K) & 4.5 & 2 & 4.5 & 2 & 4.1 & 2 & $\mathrm{mmol} / \mathrm{L}$ \\
\hline & Chloride (CL) & 115.5 & 2 & 115.5 & 2 & 116.5 & 2 & $\mathrm{mmol} / \mathrm{L}$ \\
\hline & Calcium (CA) & 10.95 & 2 & 10.65 & 2 & 10.5 & 2 & $\mathrm{mg} / \mathrm{dL}$ \\
\hline & Inorganic phosphorus (PHOS) & 5.15 & 2 & 4.95 & 2 & 4.5 & 2 & $\mathrm{mg} / \mathrm{dL}$ \\
\hline & Blood urea nitrogen (BUN) & 15.5 & 2 & 13.5 & 2 & 14.5 & 2 & $\mathrm{mg} / \mathrm{dL}$ \\
\hline & Creatinine (CREA) & 0.65 & 2 & 0.7 & 2 & 0.65 & 2 & $\mathrm{mg} / \mathrm{dL}$ \\
\hline & Glucose (GLU) & 103.5 & 2 & 101.5 & 2 & 100 & 2 & $\mathrm{mg} / \mathrm{dL}$ \\
\hline & Total protein (TPRO) & 5.7 & 2 & 5.15 & 2 & 5.25 & 2 & $\mathrm{~g} / \mathrm{dL}$ \\
\hline & Albumin (ALB) & 3.3 & 2 & 3 & 2 & 3.1 & 2 & $\mathrm{~g} / \mathrm{dL}$ \\
\hline & Globulin (GLOB) & 2.4 & 2 & 2.15 & 2 & 2.15 & 2 & $\mathrm{~g} / \mathrm{dL}$ \\
\hline & Albumin/Globulin ratio (A/G) & 1.377 & 2 & 1.395 & 2 & 1.444 & 2 & Ratio \\
\hline & Cholesterol (CHOL) & 190.5 & 2 & 152 & 2 & 161 & 2 & $\mathrm{mg} / \mathrm{dL}$ \\
\hline & Triglycerides (TRIG) & 29.5 & 2 & 30.5 & 2 & 32 & 2 & $\mathrm{mg} / \mathrm{dL}$ \\
\hline
\end{tabular}


(BUN), and mean aspartate aminotransferase activity (AST) values for the $1500 \mathrm{mg} / \mathrm{kg}$ males group were statistically higher compared to the controls. Although not statistically different, the mean $\mathrm{CL}$, mean BUN, and mean AST values for the $1500 \mathrm{mg} / \mathrm{kg}$ females were minimally higher compared to the controls. The mean total protein concentration (TPRO), mean albumin (ALB) concentration, mean albumin to globulin ratio $(\mathrm{A} / \mathrm{G})$, and mean cholesterol $(\mathrm{CHOL})$ concentration values for the $1500 \mathrm{mg} / \mathrm{kg}$ females were statistically lower compared to the controls. Although not shown to be statistically different, the mean glucose concentration (GLU) value for the $1500 \mathrm{mg} / \mathrm{kg}$ females group was lower compared to the controls (Tables 6 and 7). At Day 57 (Table 8), the mean BUN concentration value for the $300 \mathrm{mg} / \mathrm{kg}$ and $1500 \mathrm{mg} / \mathrm{kg}$ males groups was higher compared to the controls. This difference was attributed to the previous anorexia seen in the $1500 \mathrm{mg} / \mathrm{kg}$ males group, but was not of such a magnitude as to be considered an indication of renal toxicity. No urinalysis changes were reported at the highest dose $(1500 \mathrm{mg} / \mathrm{kg})$.

\section{Histology of major organs}

Dogs were euthanized the day following the end of dosing period or following a 28-day recovery period (day 57) by an overdose of sodium pentobarbital followed by exsanguination. A necropsy with tissue collection was conducted and included examination of the carcass and muscular/skeletal system, orifices, cranial cavity and external surface of the brain, neck, thoracic, abdominal and pelvic cavities associated with their organs. There were few and minimal $\alpha$-TEA-related histomorphologic or organ weight changes observed at the end of dose administration in the adrenal glands (1500 mg/ $\mathrm{kg}$ females), duodenum (1500 mg/kg males), jejunum (100 and
$1500 \mathrm{mg} / \mathrm{kg}$ males and $1500 \mathrm{mg} / \mathrm{kg}$ females), kidney, liver $(1500 \mathrm{mg} / \mathrm{kg}$, male), and thymus (300 and $1500 \mathrm{mg} /$ $\mathrm{kg}$ males and 100 and $1500 \mathrm{mg} / \mathrm{kg}$ females). These findings were considered to be incidental. No $\alpha$-TEA related macroscopic changes were observed at the end of dose administration or recovery phase, and there were no changes in organ weight. Interestingly, $\alpha$-TEA-related histomorphologic changes were noted at $1500 \mathrm{mg} / \mathrm{kg}$ on Day 29 in the duodenum (mild, diffuse villous atrophy) at $1500 \mathrm{mg} /$ $\mathrm{kg}$, jejunum (minimal, focal or multifocal dilation of a crypt) at all dose levels, kidney (mild, diffuse, bilateral vacuolation of the epithelium lining the proximal tubules) at $1500 \mathrm{mg} / \mathrm{kg}$, and liver (minimal, multifocal hepatocellular necrosis with minimal, multifocal sub-acute inflammation and moderate, diffuse vacuolation of hepatocytes) at $1500 \mathrm{mg} / \mathrm{kg}$. At the end of recovery period, minimal focal dilation of jejunal crypt and moderate diffuse vacuolation of hepatocytes was present at $1500 \mathrm{mg} / \mathrm{kg}$ and mild diffuse depletion of lymphocytes of the thymic cortex was noted at $300 \mathrm{mg} / \mathrm{kg}$.

\section{Toxicokinetics parameters}

Samples of blood were collected from the jugular vein of all animals at 1, 2, 4, 8, 24 h at day 1 and day 28 when animals received the test article. The validated LC-MS/MS method was used to analyze plasma $\alpha$-TEA concentration. Mean plasma $\alpha$-TEA concentrations in all samples obtained at $8 \mathrm{~h}$ post-dose from control animals on Day 1 were below the lower limit of quantitation $(50.0 \mathrm{ng} / \mathrm{mL})$, indicating no exposure of treated animals to $\alpha$-TEA prior to dosing. As shown in Table 9, the mean $\mathrm{T}_{\max }$ ranged from 16.0 to $24.0 \mathrm{~h}$ on Day 1 and from 6.3 to $10.7 \mathrm{~h}$ on Day 28. Elimination half-life values were not calculated or not reported on Days 1 and 28 due to insufficient data

Table 9 Mean toxicokinetic parameters at day 1 and 28-day dosing of a-TEA lysine salt

\begin{tabular}{llllllll}
\hline Day & Group & a-TEA Dose $(\mathrm{mg} / \mathrm{kg})$ & Sex & Tmax (hr) & T1/2 (hr) & Cmax (ng/mL) & AUC0-24 (hr*ng/ml) \\
\hline 1 & 2 & 100 & Male & 24 & NC & 20200 & 335000 \\
1 & 2 & 100 & Female & 20 & NC & 23900 & 35100 \\
1 & 3 & 300 & Male & 16 & NC & 41000 & 718000 \\
1 & 3 & 300 & Female & 18.7 & NC & 48600 & 72900 \\
1 & 4 & 1500 & Male & 24 & NC & 69100 & 1170000 \\
1 & 4 & 1500 & Female & 16 & NC & 76900 & 1240000 \\
28 & 2 & 100 & Male & 6.3 & NC & 148000 & 3290000 \\
28 & 2 & 100 & Female & 7 & NC & 16000 & 3620000 \\
28 & 3 & 300 & Male & 7.3 & NC & 211000 & 4740000 \\
28 & 3 & 300 & Female & 8 & NC & 212000 & 4670000 \\
29 & 4 & 1500 & Male & 8 & NC & 206000 & 4580000 \\
28 & 4 & 1500 & Female & 10.7 & NC & 192000 & 4210000 \\
\hline
\end{tabular}

Blood were collected collected at 1, 2, 4, 8, and $24 \mathrm{~h}$ following the first a-TEA administration and 2, 8, 24, 72, and 168 $\mathrm{h}$ following the last administration (day 28). The validated method (high performance liquid chromatography with mass spectrometric detection) was used to analyse plasma a-TEA concentration. $C_{\text {max }} T_{\text {max }}$ $\mathrm{T}_{1 / 2}$, AUC were determined using WinNonlin Version 6.2 software system 
points for the elimination phase. On Days 1 and 28, increases in $\mathrm{C}_{\max }$ and $\mathrm{AUC}_{0-24}$ were less than proportional for both males and females. On the first day of administration, for a 15-fold increase in dose from 100 to $1500 \mathrm{mg} /$ $\mathrm{kg}, \mathrm{C}_{\max }$ values increased 3.42-fold for males and 3.22-fold for females and $\mathrm{AUC}_{0-24}$ values increased 3.49-fold for males and 3.53-fold for females. On Day 28, $\alpha$-TEA exposure was similar at 300 and $1500 \mathrm{mg} / \mathrm{kg}$, this reflects a saturation phenomenon at $300 \mathrm{mg} / \mathrm{kg}$ dose level and above.
For a 3-fold increase in dose from 100 to $300 \mathrm{mg} / \mathrm{kg}, \mathrm{C}_{\max }$ increased 1.43-fold for males and 1.33-fold for females and $\mathrm{AUC}_{0-24}$ increased 1.44-fold for males and 1.29-fold for females. $\mathrm{C}_{\max }$ and $\mathrm{AUC}_{0-24}$ values were similar between males and females. The ratio of $\mathrm{C}_{\max }$ for males to $\mathrm{C}_{\max }$ for females at each dose level ranged from 0.84 to 0.90 on Day 1 and from 0.93 to 1.07 on Day 28. The ratio of $\mathrm{AUC}_{0-24}$ for males to $\mathrm{AUC}_{0-24}$ for females at each dose level ranged from 0.94 to 0.98 on Day 1 and from 0.91 to
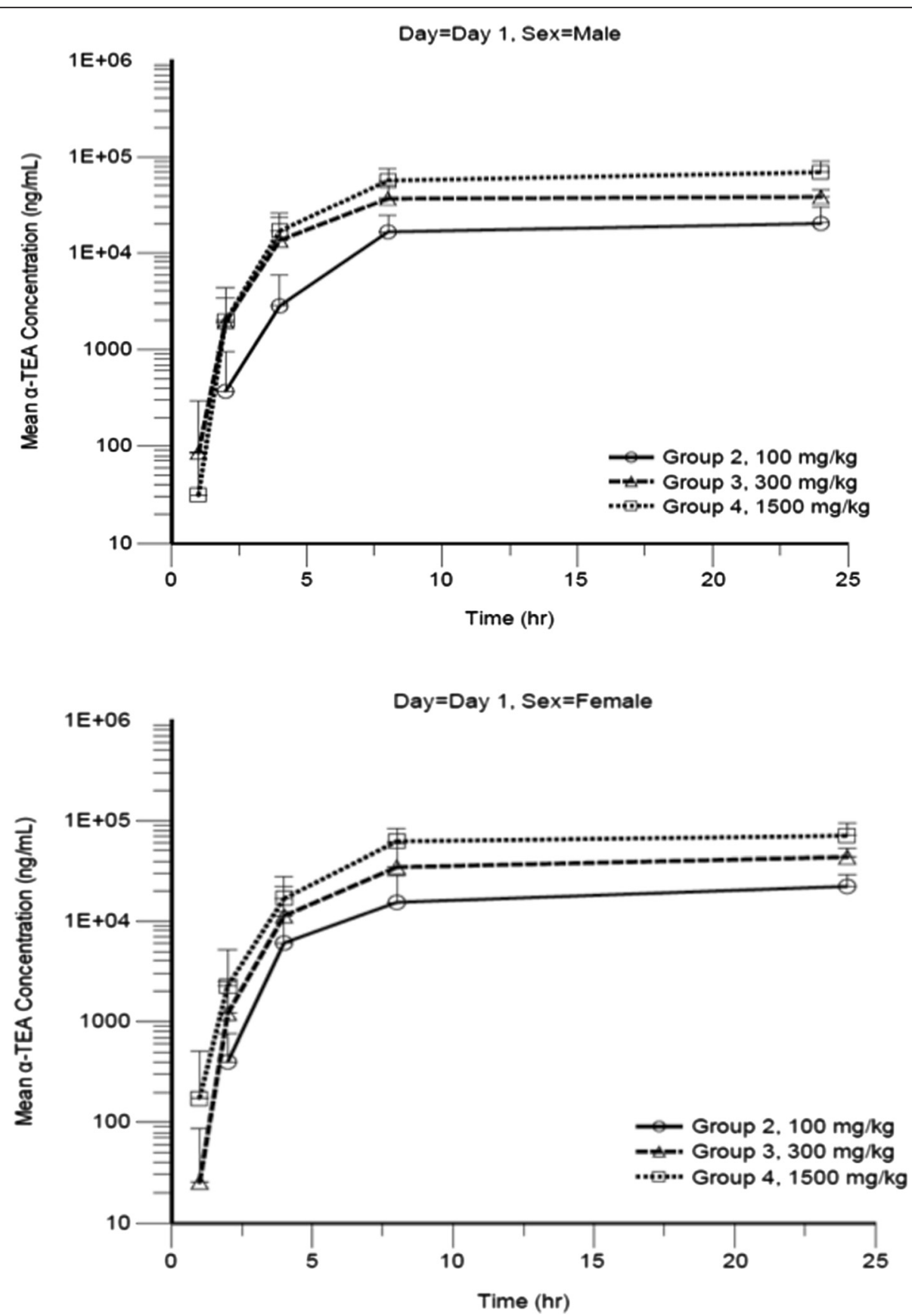

Fig. 5 Mean plasma a-TEA lysine salt concentration versus time at day 1 for male and female dogs. Male dogs received indicated doses of a-TEA lysine salt on day 1. Plots of a-TEA plasma concentration versus time are presented 
1.09 on Day 28. The AUC ratios for $\alpha$-TEA LS (ratio of $\mathrm{AUC}_{0-24}$ on Day 28 to the $\mathrm{AUC}_{0-24}$ on Day 1) was 9.82 for males and 10.31 for females at $100 \mathrm{mg} / \mathrm{kg}$ ), 6.60 for males and 6.41 for females at $300 \mathrm{mg} / \mathrm{kg}$, and 3.91 for males and 3.40 for females at $1500 \mathrm{mg} / \mathrm{kg}$. Plots of $\alpha$-TEA plasma concentration versus time are presented in Figs. 5 and 6 for Day 1 and Day 28, respectively. Exposure of animals on Day 28 was higher than exposure on Day 1, indicating accumulation of $\alpha$-TEA with multiple dosing. The extent of accumulation was greatest at the low-dose level and decreased with increasing dose, presumably due to saturation of exposure at the mid- and high-dose levels. No sex-specific differences in toxicokinetics values could be reported.

\section{Discussion}

This toxicology and pharmacokinetics study of GMP grade $\alpha$-TEA was conducted to support an Investigator New Drug (IND) application to the FDA to conduct a first-in-human clinical trial in patients with advanced
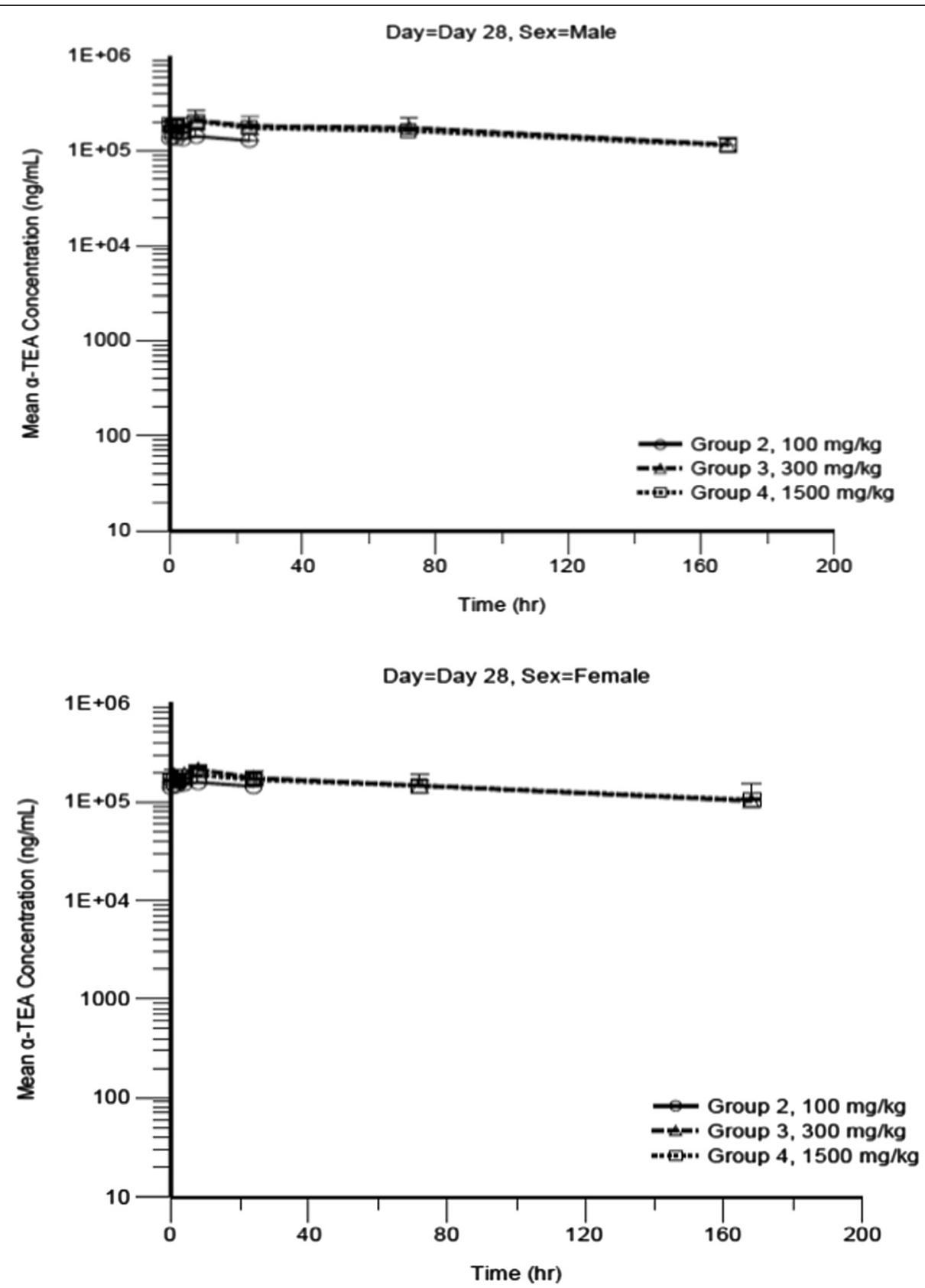

Fig. 6 Mean plasma a-TEA lysine salt concentration versus time at day 28 for male and female dogs. Female dogs received indicated doses of a-TEA lysine salt on day 1. Plots of a-TEA plasma concentration versus time are presented 
cancer based on the immunological and pro-apoptotic properties of $\alpha$-TEA. Our results demonstrate that orally administered $\alpha$-TEA LS reduces tumor growth and increases overall survival in mice bearing established tumors. This anti-tumor activity is comparable to that of $\alpha$-TEA free acid that we previously reported [11]. However, unlike $\alpha$-TEA free acid, which is difficult to manufacture for human use due to its liquid crystal properties, $\alpha$-TEA LS is crystalline in nature and can be delivered in aqueous form to animals. In the non-rodent dose escalation study, in which optimal conditions and timing of orally administered $\alpha$-TEA LS were assessed, there were no signs of toxicity in dogs that received daily doses of up to $1500 \mathrm{mg} / \mathrm{kg}$. No moribundity or mortality was observed at any of the dose levels tested throughout the treatment period. No genderrelated differences were observed. Minor histomorphologic alterations were observed at the highest dose but these changes were mostly reversed at the end of the recovery period.

Clinical pathology changes were limited to increased white blood cell (lymphocyte and neutrophil) counts, and fibrinogen levels at $1500 \mathrm{mg} / \mathrm{kg}$; these changes were likely related to mild inflammation and/or stress but all these findings were resolved by the end of recovery period. Although a full and complete toxicokinetic profile could not be determined from the study, there was an apparent less than dose proportional increase in AUC over the duration of the study. The extent of accumulation was greatest at the low-dose level and decreased with increasing dose, presumably due to saturation of exposure at the mid- and high-dose levels.

\section{Conclusion}

In summary, in this comprehensive examination of the prolonged use of GMP-grade $\alpha$-TEA LS in dogs, followed by a 28-day recovery period, we report absence of any dose limiting toxicity. Our findings suggest that $\alpha$-TEA is safe and tolerated and determined that the highest nonseverely toxic dose (HNSTD) to be $1500 \mathrm{mg} / \mathrm{kg}$. These findings formed the basis for initiating a first-in-human clinical trial of $\alpha$-TEA in patients with advanced cancer, which is ongoing.

\section{Abbreviations}

a-TEA: Alpha-Tocopheryloxyacetic free acid; a-TEA LS: a-TEA Lysine Salt; HPLC: High Performance Liquid Chromatography.

\section{Competing interests}

The authors declare that they have no competing interests.

\section{Authors' contributions}

ETA, BC, WU, conception and designed research; TH planned research, ZA performed research; ETA, BC, WU analyzed data; BSG wrote the manuscript with contribution from the coauthors. All authors read and approved the final manuscript.

\section{Acknowledgements}

This study was supported by funds from The Safeway Foundation and Providence Cancer Center.

Received: 10 October 2015 Accepted: 28 February 2016

Published online: 08 March 2016

\section{References}

1. Lawson KA, Anderson K, Menchaca M, Atkinson J, Sun L, Knight V, Gilbert BE, Conti C, Sanders BG, Kline K. Novel vitamin E analogue decreases syngeneic mouse mammary tumor burden and reduces lung metastasis. Mol Cancer Ther. 2003;2(5):437-44.

2. Neuzil J, Dong LF, Ramanathapuram L, Hahn T, Chladova M, Wang XF, Zobalova R, Prochazka L, Gold M, Freeman R, et al. Vitamin E analogues as a novel group of mitocans: Anti-cancer agents that act by targeting mitochondria. Mol Aspects Med. 2007;28(5-6):607-45.

3. Hahn T, Akporiaye ET. alpha-TEA as a stimulator of tumor autophagy and enhancer of antigen cross-presentation. Autophagy. 2013;9(3):429-31.

4. Hahn T, Bradley-Dunlop DJ, Hurley LH, Von-Hoff D, Gately S, Mary DL, Lu H, Penichet ML, Besselsen DG, Cole BB, et al. The vitamin E analog, alphatocopheryloxyacetic acid enhances the anti-tumor activity of trastuzumab against HER2/neu-expressing breast cancer. BMC Cancer. 2011;11:471.

5. Hahn T, Jagadish B, Mash EA, Garrison K, Akporiaye ET. alpha-Tocopheryloxyacetic acid: a novel chemotherapeutic that stimulates the antitumor immune response. Breast Cancer Res. 2011;13(1):R4.

6. Li Y, Hahn T, Garrison K, Cui ZH, Thorburn A, Thorburn J, Hu HM, Akporiaye $E T$. The vitamin E analogue alpha-TEA stimulates tumor autophagy and enhances antigen cross-presentation. Cancer Res. 2012;72(14):3535-45.

7. Lawson KA, Anderson K, Snyder RM, Simmons-Menchaca M, Atkinson J, Sun LZ, Bandyopadhyay A, Knight V, Gilbert BE, Sanders BG, et al. Novel vitamin $\mathrm{E}$ analogue and 9-nitro-camptothecin administered as liposome aerosols decrease syngeneic mouse mammary tumor burden and inhibit metastasis. Cancer Chemother Pharmacol. 2004;54(5):421-31.

8. Dong LF, Grant G, Massa H, Zobalova R, Akporiaye E, Neuzil J. alphaTocopheryloxyacetic acid is superior to alpha-tocopheryl succinate in suppressing HER2-high breast carcinomas due to its higher stability. Int J Cancer. 2012;131(5):1052-8.

9. Hahn T, Szabo L, Gold M, Ramanathapuram L, Hurley LH, Akporiaye ET. Dietary administration of the proapoptotic vitamin E analogue alphatocopheryloxyacetic acid inhibits metastatic murine breast cancer. Cancer Res. 2006;66(19):9374-8.

10. Hahn T, Fried K, Hurley LH, Akporiaye ET. Orally active alpha-tocopheryloxyacetic acid suppresses tumor growth and multiplicity of spontaneous murine breast cancer. Mol Cancer Ther. 2009;8(6):1570-8.

11. Hahn T, Akporiaye ET. Repeat dose study of the novel proapoptotic chemotherapeutic agent alpha-tocopheryloxy acetic acid in mice. Anticancer Drugs. 2012;23(4):455-64.

Submit your next manuscript to BioMed Central and we will help you at every step:

- We accept pre-submission inquiries

- Our selector tool helps you to find the most relevant journal

- We provide round the clock customer support

- Convenient online submission

- Thorough peer review

- Inclusion in PubMed and all major indexing services

- Maximum visibility for your research 